\title{
SINGULARITIES OF THE SCATTERING KERNEL AND SCATTERING INVARIANTS FOR SEVERAL STRICTLY CONVEX OBSTACLES
}

\author{
VESSELIN M. PETKOV AND LUCHEZAR N. STOJANOV
}

\begin{abstract}
Let $\Omega \subset \mathbf{R}^{n}$ be a domain such that $\mathbf{R}^{n} \backslash \Omega$ is a disjoint union of a finite number of compact strictly convex obstacles with $C^{\infty}$ smooth boundaries. In this paper the singularities of the scattering kernel $s(t, \theta, \omega)$, related to the wave equation in $\mathbf{R} \times \Omega$ with Dirichlet boundary condition, are studied. It is proved that for every $\omega \in S^{n-1}$ there exists a residual subset $\mathscr{R}(\omega)$ of $S^{n-1}$ such that for each $\theta \in \mathscr{R}(\omega), \theta \neq \omega$,

$$
\text { singsupp } s(t, \theta, \omega)=\left\{-T_{\gamma}\right\}_{\gamma},
$$

where $\gamma$ runs over the scattering rays in $\Omega$ with incoming direction $\omega$ and with outgoing direction $\theta$ having no segments tangent to $\partial \Omega$, and $T_{\gamma}$ is the sojourn time of $\gamma$. Under some condition on $\Omega$, introduced by $M$. Ikawa, the asymptotic behavior of the sojourn times of the scattering rays related to a given configuration, as well as the precise rate of the decay of the coefficients of the main singularity of $s(t, \theta, \omega)$, is examined.
\end{abstract}

\section{INTRODUCTION}

Let $\Omega \subset \mathbf{R}^{n}, n \geq 3, n$ odd, be an open domain with $C^{\infty}$ smooth boundary $\partial \Omega$ and bounded complement $K=\mathbf{R}^{n} \backslash \Omega \subset\left\{x:|x| \leq \rho_{0}\right\}$. The scattering operator $S$ for the wave equation in $\mathbf{R} \times \Omega$ with Dirichlet boundary condition is a unitary operator from $L^{2}\left(\mathbf{R} \times S^{n-1}\right)$ into $L^{2}\left(\mathbf{R} \times S^{n-1}\right)$ and the kernel $s\left(t-t^{\prime}, \theta, \omega\right)$ of $S$-Id is called the scattering kernel (see [10, 11]). For fixed $(\theta, \omega) \in S^{n-1} \times S^{n-1}$ we have $s(t, \theta, \omega) \in \mathscr{S}^{\prime}(\mathbf{R})$ and

$$
s(t, \theta, \omega)=C_{n} \int_{\partial K} \partial_{\tau}^{n-2} \partial_{\nu} w(\langle x, \theta\rangle-t, x ; \omega) d S_{x} .
$$

Here $w(\tau, x ; \omega)$ is the solution to the problem

$$
\left\{\begin{array}{l}
\left(\partial_{\tau}^{2}-\Delta_{x}\right) w=0 \text { in } \mathbf{R} \times \Omega, \\
w=0 \text { on } \mathbf{R} \times \partial \boldsymbol{\Omega}, \\
\left.w\right|_{\tau<-\rho_{0}}=\delta(\tau-\langle x, \omega\rangle),
\end{array}\right.
$$

Received by the editors October 28, 1987.

1980 Mathematics Subject Classification (1985 Revision). Primary 35P25, 35R30; Secondary $34 \mathrm{C} 35$.

This work was partially supported by the Bulgarian Committee of Sciences, Grant No. 52 (1987) to V. M. P. and Grant No. 44 (1987) to L. N. S. 
$\nu$ is the interior unit normal to $\partial \Omega$ (pointing into $\Omega$ ),

$$
C_{n}=(-1)^{(n+1) / 2} 2^{-n} \pi^{(1-n)}
$$

and $d S_{x}$ is the induced measure on $\partial K$. The integral (1.1) is interpreted in the sense of distributions (see $[11,26])$.

Denoting by $\hat{s}(\lambda, \theta, \omega)$ the Fourier transform of $s(t, \theta, \omega)$ with respect to $t$,

$$
a(\lambda, \theta, \omega)=(2 \pi / i \lambda)^{(n-1) / 2} \hat{s}(\lambda, \theta, \omega)
$$

is called the scattering amplitude. The analysis of the asymptotic behavior of $a(\lambda, \theta, \omega)$ as $\lambda \rightarrow \infty$ is closely related to the examination of the singularities of $s(t, \theta, \omega)$.

For $\omega \neq \theta$ and for strictly convex obstacles $s(t, \theta, \omega)$ has only one singularity related to the reflecting ray at $\hat{x} \in \partial K, \hat{x}$ being the point where $\nu(\hat{x})=(\theta-\omega) /\|\theta-\omega\|$. On the other hand, for nonconvex obstacles there exists $\omega \in S^{n-1}$ for which $s(t,-\omega, \omega)$ has at least two different singularities $[26,29]$.)

For obstacles with arbitrary geometry $\max _{t} \operatorname{singsupp} s(t, \theta, \omega)$ has been studied in $[11,17,27]$. One of the authors investigated in [16] the set of all singularities of $s(t, \theta, \omega)$ for nonconvex obstacles, making some restrictions on the geometry of the rays incoming with direction $\omega$. These restrictions are too difficult for verification. Nevertheless, some of them are fulfilled for generic obstacles, provided $\omega, \theta$ are fixed (see $[20,21]$ ).

As suggested in $[5,16]$, singsupp $s(-t, \theta, \omega)$ must be related to the sojourn times of the so-called $(\omega, \theta)$-rays (see $\S 2$ for a precise definition). It is natural to expect that for generic directions $(\omega, \theta)$ the sojourn times of all ordinary $(\omega, \theta)$-rays belong to singsupp $s(-t, \theta, \omega)$. A $(\omega, \theta)$-ray $\gamma$ is called ordinary if $\gamma$ has no segments tangent to $\partial K$. In general a nonconvex obstacle $K$ could admit (generalized) rays with incoming direction $\omega$ and outgoing direction $\theta$ which have some gliding segments on $\partial K$. This leads to considerable difficulties when we try to prove the above assertion for nonconvex obstacles.

Throughout this paper we assume

$$
\left[\begin{array}{l}
K=\bigcup_{i=1}^{s} K_{i}, K_{i} \cap K_{j}=\varnothing \text { for } i \neq j \\
K_{i} \quad \text { are compact and strictly convex for } i=1, \ldots, s .
\end{array}\right.
$$

For fixed $\omega \neq \theta$, let $\mathscr{L}_{\omega, \theta}$ be the set of all ordinary $(\omega, \theta)$-rays with reflections on $\partial K$. Given $\gamma \in \mathscr{L}_{\omega, \theta}^{\omega}$, denote by $x_{\gamma}$ (resp. by $y_{\gamma}$ ) the first (resp. the last) reflection point of $\gamma$. Let $Z_{\omega}$ be a fixed hyperplane so that $K$ is contained in the open half-space $H_{\omega}$ determined by $Z_{\omega}$ and having $\omega$ as an inward normal. If $\gamma$ hits $Z_{\omega}$ at $A_{\gamma}$, then in some neighborhood $U_{\gamma}$ of $A_{\gamma}$ we can define the map $J_{\gamma}: U_{\gamma} \ni u \rightarrow \theta(u), \theta(u)$ being the outgoing direction of the $(\omega, \theta(u))$-ray issued from $u$ (see $[5,16]$ and $\S 3$ ). Denote by $T_{\gamma}$ the sojourn time of $\gamma$ (see $[5,16]$ and $\S 4$ for the definition). Finally, recall that a subset 
$\mathscr{R} \subset S^{n-1}$ is called residual if $\mathscr{R}$ is a countable intersection of open and dense subsets of $S^{n-1}$. Our main result is the following.

Theorem 1.1. Let $\omega \in S^{n-1}$ be fixed. Then there exists a residual subset $\mathscr{R}(\omega) \subset S^{n-1}$ such that for each $\theta \in \mathscr{R}(\omega), \theta \neq \omega$, we have

$$
\operatorname{singsupp} s(t, \theta, \omega)=\left\{-T_{\gamma}: \gamma \in \mathscr{L}_{\omega, \theta}\right\} \text {. }
$$

Moreover, near $-T_{\gamma}$ we have

$$
\begin{aligned}
s(t, \theta, \omega)= & (2 \pi)^{(1-n) / 2}(-1)^{m_{\gamma}-1} i^{\sigma_{\gamma}} \\
& \times\left|\frac{\operatorname{det} d J_{\gamma}\left(A_{\gamma}\right)\left\langle\nu\left(x_{\gamma}\right), \omega\right\rangle}{\left\langle\nu\left(y_{\gamma}\right), \theta\right\rangle}\right|^{-1 / 2} \delta^{(n-1) / 2}\left(t+T_{\gamma}\right)
\end{aligned}
$$

+ smoother terms. Here $m_{\gamma}$ is the number of reflections of $\gamma, \sigma_{\gamma} \in \mathbf{N}$ is a Maslov index, and $\langle$,$\rangle is the inner product in \mathbf{R}^{n}$.

Equality (1.4) is similar to the Poisson relation for the distribution $\sigma(t)=$ $\sum_{j} \cos \lambda_{j} t$, where $\left\{\lambda_{j}^{2}\right\}_{j=1}^{\infty}$ are the eigenvalues of the Laplace-Beltrami operator on a compact manifold without boundary [3] or the eigenvalues of the Dirichlet problem for the Laplacian in a bounded domain $\mathscr{O} \subset \mathbf{R}^{2}$ (see $\left.[19,22]\right)$. In these cases the singularities of $\sigma(t)$ are included in the union of the lengths of all periodic geodesics. For this reason we will call $\left\{T_{\gamma}: \gamma \in \mathscr{L}_{\omega, \theta}\right\}$ the scattering length spectrum of $K$ related to $\omega, \theta$. Nakamura and Soga [15] established (1.4) for $\theta=-\omega$ and for two disjoint balls $\mathscr{O}_{i}, i=1,2$, making some restrictions on $\operatorname{dist}\left(\mathscr{O}_{1}, \mathscr{O}_{2}\right)$ and the diameters of $\mathscr{O}_{i}, i=1,2$.

Formula (1.5) was obtained in [16]. Similar results concerning the singularities of $\sigma(t)$ when $t$ coincides with the period of some periodic geodesic have been proved in [3,6]. Theorem 1.1 says that for fixed $\omega \in S^{n-1}$ and $\theta \in \mathscr{R}(\theta)$, $\theta \neq \omega$, from the singularities of $s(t, \theta, \omega)$ we can recover as scattering data all sojourn times $T_{\gamma}, \gamma \in \mathscr{L}_{\omega, \theta}$ together with the corresponding coefficients $c_{\gamma}$ in front of $\delta^{(n-1) / 2}\left(t+T_{\gamma}\right)$. On the other hand, from the scattering kernel we cannot determine when $\theta$ belongs to $\mathscr{R}(\omega)$.

The proof of Theorem 1.1 is based on three main points. First, we study the topological properties of the map $J_{\alpha}$ related to a fixed configuration $\alpha$. We describe the maximal subset $M_{\alpha} \subset \stackrel{\alpha}{Z}_{\omega}$, where $J_{\alpha}: M_{\alpha} \rightarrow J_{\alpha}\left(M_{\alpha}\right)$ becomes a homeomorphism. This leads to the uniqueness of the $(\omega, \theta)$-ray associated to $\alpha$. Second, for generic $\theta$ we establish two properties of $(\omega, \theta)$-rays. One of them says that the sojourn times of different $(\omega, \theta)$-rays are different too. Third, to examine singsupp $s(t, \theta, \omega) \cap[-T, T]$, we introduce a special localization of the problem (1.2) different from those previously used in $[16,17,27]$. This localization depends on $T$ as well as on the results in $\S \S 3$ and 4 playing a crucial role when we deal with the rays admitting tangent segments. Moreover, our localization enables us to eliminate completely the investigation of the mixed problems with data localized in the shadow domain with respect to $\omega$ (see [16]). 
The results of Melrose and Sjöstrand [13,14] imply that for nontrapping obstacles the sojourn times $T_{\gamma}$ of all $(\omega, \theta)$-rays $\gamma$ are uniformly bounded for all $(\omega, \theta) \in S^{n-1}$. For trapping obstacles we expect that for suitably chosen $\omega, \theta$ we have

$$
\sup \left\{T_{\gamma}: \gamma \in \mathscr{L}_{\omega, \theta}\right\}=\infty .
$$

In our case we are able to establish (1.6) making the additional assumption

$$
\left[\begin{array}{l}
\text { For all triples }\left(i_{1}, i_{2}, i_{3}\right) \in\{1, \ldots, s\}^{3}, i_{j} \neq i_{k}, j \neq k, \\
\text { the convex hull of } K_{i_{1}} \cup K_{i_{2}} \text { has no common points with } K_{i_{3}} .
\end{array}\right.
$$

This condition was introduced by Ikawa in [9], where he proved that for each configuration

$$
\alpha=\left(i_{1}, \ldots, i_{k}\right), \quad i_{1} \neq i_{k},
$$

there exists a unique periodic ray $\gamma_{\alpha}$ with successive reflection points $\tilde{x}_{j} \in \partial K_{i_{j}}$, $j=1, \ldots, k$. Under condition (H) we show in $\S 6$ that for each such $\alpha$, choosing $\omega, \theta$, suitably for every $q \geq 0$ there exists an ordinary $(\omega, \theta)$-ray $\gamma_{m}^{1 l}$ with $m=q k+l$ reflection points

$$
x_{p k+j} \in \partial K_{i_{j}}, \quad 0 \leq p \leq q, 1 \leq j \leq k(1 \leq j \leq l \text { for } p=q) .
$$

The choice of $\omega$ and $\theta$ depends on some condition of visibility concerning $K_{i_{1}}$ and $K_{i_{1}}$ which we are able to arrange exploiting $(\mathrm{H})$. On the other hand, to guarantee that $\gamma_{m}^{1 l}$ are ordinary rays, we apply essentially our results in $\S 4$.

In our paper we study the asymptotic behavior of the sojourn times $T_{q k+l}^{1 l}$ of $\gamma_{q k+l}^{1 l}$ as $q \rightarrow \infty$. The leading term of the corresponding asymptotic is merely $q d_{\alpha}, d_{\alpha}$ being the length of $\gamma_{\alpha}$ (see Theorem 6.5). Consequently, the scattering length spectrum $\left\{\mathscr{L}_{\omega, \theta}: \omega \neq \theta\right\}$ determines the lengths of all periodic rays $\gamma_{\alpha}$. For this reason we could consider $d_{\alpha}$ as scattering invariants. In our previous paper [22] we proved that the lengths of $\gamma_{\alpha}$ can be recovered from the singularities of the distribution $\sum_{j} e^{i t \bar{\lambda}_{j}}, \tilde{\lambda}_{j}$ being the poles of the scattering matrix, provided that $K$ is a generic obstacle. The above result says that we may obtain the same information from the scattering length spectrum.

Marvizi and Melrose [12] and Colin de Verdiére [2] studied the asymptotic behavior of the lengths of periodic geodesics for strictly convex planar regions $\mathscr{O} \subset \mathbf{R}^{2}$. In [12] these geodesics approximate the boundary $\partial \mathscr{O}$, while in [2] they approximate an elliptic periodic ray. In our situation $\gamma_{\alpha}$ is a hyperbolic ray and in the asymptotic of $T_{q k+l}^{1 l}$ we obtain two terms modulo a remainder $O\left(\delta^{q}\right)$ with $0<\delta<1$. For two disjoint balls some partial results have been obtained in [15].

In $\S 7$ we examine the precise rate of decay of the coefficients $c_{m}^{1 l}$ in front of $\delta^{(n-1) / 2}\left(t+T_{m}^{1 l}\right)$ as $m \rightarrow \infty$. We obtain the asymptotic behavior of $\ln \left|c_{q k+l}^{1 l}\right|$ 
as $q \rightarrow \infty$ with leading term

$$
q c_{\alpha}=-\frac{1}{2} q \sum_{j=1}^{n-1} \ln \left|\mu_{j}\right|
$$

Here $\mu_{j},\left|\mu_{j}\right|>1$, are the eigenvalues of the (linear) Poincare map related to $\gamma_{\alpha}$. Our analysis is based on the representation of the Poincare map [18, 22] which makes it possible to simplify the calculation of $\operatorname{det} d J_{\gamma}\left(A_{\gamma}\right)$ and the examination of the asymptotic of $\ln \left|c_{q k+l}^{1 l}\right|$.

For two obstacles $K_{i}, i=1,2$, condition $(\mathrm{H})$ is trivial. Then from the scattering length spectrum we can recover the invariants $d=\operatorname{dist}\left(K_{1}, K_{2}\right)$ and $c_{d}=-\sum_{j=1}^{n-1} \ln \left|\mu_{j}\right|$ related to the unique periodic ray. Moreover, we may determine the first sequence

$$
\lambda_{j}=-\left(i c_{d}\right) / d+j(d / \pi), \quad j \in Z,
$$

of pseudopoles of the scattering matrix (see $[1,4,7])$. In this case $\lambda_{j}$ are connected with the meromorphic continuation of the leading term

$$
a_{0}(\lambda)=\sum_{\gamma \in \mathscr{L}_{\omega, \theta}} c_{\gamma} e^{-i \lambda T_{\gamma}}
$$

of the scattering amplitude $a(-\lambda, \theta, \omega)$. It is interesting to study the analytic properties of $a_{0}(\lambda)$ for more than two obstacles.

The paper is organized as follows. In $\S 2$ some notation and preliminary facts are given. In $\S 3$ we study the map $J_{\alpha}$, while in $\S 4$ we treat the $(\omega, \theta)$-rays for generic $\theta . \S 5$ is devoted to the proof of Theorem 1.1. In $\S 6$ we discuss the existence of $(\omega, \theta)$-rays and property (1.6) together with the asymptotic of $T_{q k+l}^{1 l}$. Finally, the asymptotic of $\ln \left|c_{q k+l}^{1 l}\right|$ is examined in $\S 7$. Part of our results have been announced in [23].

\section{Preliminaries}

2.1. Let $l_{1}$ and $l_{2}$ be linear segments with a common end point $x \in \partial K$. We say that $l_{1}$ and $l_{2}$ satisfy the law of reflection at $x$ with respect to $\partial K$ if either $l_{1}$ and $l_{2}$ lie on a common line tangent to $\partial K$ at $x$ and $l_{1} \cap l_{2}=\{x\}$ (the case of tangency) or $l_{1}$ and $l_{2}$ make equal acute angles with $\nu(x)$ lying in a common two-dimensional plane with $\nu(x)$ (the case of a proper reflection).

Let $\omega, \theta \in S^{n-1}$ and let $\gamma=\bigcup_{i=0}^{k} l_{i}$ be a curve in $\mathbf{R}^{n}$, where $l_{i}=\left[x_{i}, x_{i+1}\right]$ are finite segments for $i=1, \ldots, k-1(k \geq 1), x_{i} \in \partial K$ for $0 \leq i \leq k$, $l_{0}$ (resp. $l_{k}$ ) is an infinite segment starting at $x_{1}$ (resp. at $x_{k}$ ) and having direction $-\omega$ (resp. $\theta)$. Then $\gamma$ will be called an $(\omega, \theta)$-ray if the following conditions hold:

(i) the open segments $\stackrel{\circ}{l}_{i}, i=0, \ldots, k$, have no common points with $K$, and

(ii) for every $i=0, \ldots, k-1$ the segments $l_{i}$ and $l_{i+1}$ satisfy the law of reflection at $x_{i+1}$ with respect to $\partial K$. 
Points $x_{1}, \ldots, x_{k}$ will be called reflection points of $\gamma$. Some of them are proper reflection points, and if $x_{i}$ is among the others, then we say that $\gamma$ is tangent to $\partial K$ at $x_{i}$. This definition of an $(\omega, \theta)$-ray is slightly different from that in $[20,21]$. If all reflection points of $\gamma$ are proper ones, then $\gamma$ will be called an ordinary $(\omega, \theta)$-ray.

2.2. Given $\omega \in S^{n-1}$, let $Z_{\omega}$ be a fixed hyperplane so that $K$ is contained in the open half-space $H_{\omega}$ determined by $Z_{\omega}$ and having $\omega$ as an inward normal.

For $u \in Z_{\omega}$ consider the motion of a point with unit velocity starting at $u$ with direction $\omega$ and reflecting from $\partial K$ following the usual reflection law or tangent to $\partial K$. By $S_{t}(u)$ we denote the shift of $u$ at time $t \geq 0$, and by $N_{t}(u)$ we denote the velocity vector of the point at the moment $t$ (for $y=S_{t}(u) \in \partial K$ we identify $N_{t}(u)$ with $\sigma_{y}\left(N_{t}(u)\right), \sigma_{y}$ being the symmetry with respect to the tangent plane to $\partial K$ at $y)$. By $x_{1}(u), x_{2}(u), \ldots$ we denote the successive reflection points (proper or not) of the ray $\gamma(u)=\left\{S_{t}(u): t \geq 0\right\}$. By $t_{1}(u), t_{2}(u), \ldots$ we denote the times of the corresponding reflections. Clearly, these two sequences may be empty, finite, or infinite. By $r(u)$ we denote the number of all reflections of $\gamma(u)$. Thus we have $r(u)=0,1, \ldots, \infty$.

The following property of $S_{t}(u)$ is well known in the theory of dispersing billiards (see [24, 25, 28]).

Proposition 2.1. Let $U$ be an open subset of $Z_{\omega}$ and let $t>0$ be such that for every $u \in U$ we have $r(u) \geq m, t_{m}(u)<t<t_{m+1}(u)$, and $x_{1}(u), \ldots, x_{m}(u)$ are proper reflection points of $\gamma(u)$. Assume $x_{j}(u) \in \partial K_{i_{j}}$ for every $u \in U$ and $j=1, \ldots, m$. Then $S_{t}(u)$ is a smooth surface in $\mathbf{R}^{n}$ and the second fundamental form of $S_{t}(u)$ with respect to the normal field $\left\{N_{t}(u): u \in U\right\}$ is positive definite.

2.3. In this paper smooth means $C^{\infty}$. For a subset $Y$ of a topological space $X$ by $\bar{Y}(\stackrel{\circ}{Y})$ we denote the closure (interior) of $Y$ in $X$. We will use the notation

$$
\partial K_{i}^{ \pm}=\left\{y \in \partial K_{i}:\langle\nu(y), \omega\rangle \lessgtr 0\right\} .
$$

2.4. Let $A$ be a real $(p \times p)$ matrix and let $I$ be the identity $(p \times p)$ matrix. Recall that

$$
|\operatorname{det} A| \leq(1+\|A-I\|)^{p},
$$

$\|A\|$ being the norm of the operator $A: \mathbf{C}^{p} \rightarrow \mathbf{C}^{p}$. If $M$ is a symmetric and positive definite matrix, then for $\lambda>0$ we have

$$
\left\|(I+\lambda M)^{-1}\right\| \leq(1+\lambda \sigma)^{-1}, \quad\left\|M(I+\lambda M)^{-1}\right\| \leq 1 / \lambda,
$$

where $\sigma=\min \operatorname{spec} M, \operatorname{spec} M$ being the spectrum of $M$. 


\section{THE MAPS $J_{\alpha}$}

By a configuration $\alpha$ with length $|\alpha|=m, m \geq 1$, we mean a symbol $\alpha=\left(i_{1}, i_{2}, \ldots, i_{m}\right)$ such that $i_{j} \in\{1,2, \ldots, s\}$ for all $j$ and $i_{j} \neq i_{j+1}$ for $j=1,2, \ldots, m-1$.

Let $\omega \in S^{n-1}$ and let $Z_{\omega}$ be as in subsection 2.2. Consider the sets

$$
\begin{aligned}
& F_{\alpha}=\left\{u \in Z_{\omega}: r(u) \geq m \text { and } x_{j}(u) \in \partial K_{i_{j}} \text { for } j=1, \ldots, m\right\}, \\
& U_{\alpha}=\left\{u \in F_{\alpha}: x_{j}(u) \text { is a proper reflection point for all } j \leq m\right\} .
\end{aligned}
$$

Define the map

$$
J_{\alpha}: F_{\alpha} \rightarrow S^{n-1}
$$

by $J_{\alpha}(u)=N_{t}(u)$ for arbitrary $t$ with $t_{m}(u)<t<t_{m+1}(u)$ (resp. $t<\infty$ if $r(u)=m)$. This map was introduced by Guillemin [5] in a slightly different context.

In this section we establish some properties of the maps $J_{\alpha}$ which will be used in the next sections.

Lemma 3.1.

(a) For every $u \in \overline{F_{\alpha}}$ there exists a configuration $\beta=\left(\bar{i}_{1}, \ldots, \bar{i}_{p}\right)$ with $p \geq m, u \in F_{\beta}$ such that there is a sequence $p_{1}<p_{2}<\cdots<p_{m}=p$ with $\overline{i_{p_{j}}}=i_{j}$ for all $j=1, \ldots, m$. Moreover, if $x_{r}(u)$ is a proper reflection point of $\gamma(u)$ for some $r=1, \ldots, p$, then $r=p_{j}$ for some $j=1, \ldots, m$;

(b) $J_{\alpha}$ can be extended to a continuous map $J_{\alpha}: \overline{F_{\alpha}} \rightarrow S^{n-1}$;

(c) $\stackrel{\circ}{F}_{\alpha}=U_{\alpha}$.

Proof. The proofs of (a) and (b) are quite elementary and we omit them. To establish (c), first note that $U_{\alpha}$ is obviously open and $U_{\alpha} \subset F_{\alpha}$. Take $u \in \stackrel{\circ}{F}_{\alpha}$ and suppose $u \notin U_{\alpha}$. Then $\gamma(u)$ is tangent to $\partial K$ at $x_{j}(u)$ for some $j=$ $1, \ldots, m$. Let $j$ be the minimal number with this property and for convenience set $x_{0}(u)=u$ and $t_{0}(u)=0$. Choose an arbitrary $t$ with $t_{j-1}(u)<t<t_{j}(u)$ and an open neighborhood $U$ of $u$ in $\stackrel{\circ}{F}_{\alpha}$ so small that $t_{j-1}\left(u^{\prime}\right)<t<t_{j}\left(u^{\prime}\right)$ for all $u^{\prime} \in U$. We may arrange $S_{t}(U)$ to be strictly convex with respect to the normal field $\left\{N_{t}\left(u^{\prime}\right): u^{\prime} \in U\right\}$ (cf. subsection 2.2 and Proposition 2.1). A simple geometric argument shows that there exists $u^{\prime} \in U$ so that the straight ray starting at $x_{j-1}\left(u^{\prime}\right)$ with direction $N_{t}\left(u^{\prime}\right)$ has no common points with $\partial K_{i_{j}}$. Thus $u^{\prime} \notin F_{\alpha}$, which gives a contradiction. Therefore $\stackrel{\circ}{F}_{\alpha} \subset U_{\alpha}$ and we obtain (c).

Consider the set

$$
L_{\alpha}=\left\{u \in \overline{F_{\alpha}}: N_{t}(u)=\omega \text { for every } t \geq 0\right\} .
$$


For $u \in L_{\alpha}$ the ray $\gamma(u)$ goes straightforward with constant direction $\omega$ and has common (tangent) points with $\partial K_{i_{1}}, \ldots, \partial K_{i_{m}}$ and, possibly, with some other $\partial K_{r}$. Clearly, $L_{\alpha}$ is compact, $\stackrel{\circ}{\alpha}_{\alpha}=\varnothing$, and $L_{\alpha} \cap U_{\alpha}=\varnothing$. In fact, $L_{\alpha}$ is contained in the boundary (in $Z_{\omega}$ ) of the orthogonal projection of $K_{i_{1}}$ onto $Z_{\omega}$. Consequently, the set

$$
M_{\alpha}=\overline{F_{\alpha}} \backslash L_{\alpha}
$$

contains $\stackrel{\circ}{F_{\alpha}}$.

Since $J_{\alpha}: \overline{F_{\alpha}} \rightarrow S^{n-1}$ is continuous and $\overline{F_{\alpha}}$ is compact,

$$
E_{\alpha}=J_{\alpha}\left(\overline{F_{\alpha}}\right)
$$

is a compact subset of $S^{n-1}$. Note that $J_{\alpha}\left(L_{\alpha}\right)=\{\omega\}$, so $J_{\alpha}\left(M_{\alpha}\right)$ is either $E_{\alpha}$ or $E_{\alpha} \backslash\{\omega\}$.

The main result in this section is the following.

Theorem 3.2. For every configuration $\alpha$ the map

$$
J_{\alpha}: M_{\alpha} \rightarrow J_{\alpha}\left(M_{\alpha}\right)
$$

is a homeomorphism.

Proof. It is sufficient to prove that if $u \in M_{\alpha}, v \in \overline{F_{\alpha}}$ with $u \neq v$, then $J_{\alpha}(u) \neq$ $J_{\alpha}(v)$. Indeed, assume this true. Then $J_{\alpha}: M_{\alpha} \rightarrow J_{\alpha}\left(M_{\alpha}\right)$ is a continuous bijection and it remains to show that $J_{\alpha}^{-1}$ is continuous. Take a sequence $\left\{u_{k}\right\} \subset M_{\alpha}$ and $u \in M_{\alpha}$ so that $J_{\alpha}\left(u_{k}\right) \rightarrow J_{\alpha}(u)$. Let $v$ be a cluster point of $\left\{u_{k}\right\}$; then $v \in \overline{F_{\alpha}}$ and clearly $J_{\alpha}(v)=J_{\alpha}(u)$. Therefore $v=u$, and this shows that $u$ is the only cluster point of $\left\{u_{k}\right\}$ in $\overline{F_{\alpha}}$. Thus $u_{k} \rightarrow u$ and $J_{\alpha}: M_{\alpha} \rightarrow J_{\alpha}\left(M_{\alpha}\right)$ is a homeomorphism.

Let $\alpha=\left(i_{1}, \ldots, i_{m}\right)$ and let $u \neq v$ be elements of $M_{\alpha}$ and $\overline{F_{\alpha}}$, respectively. By Lemma $3.1(\mathrm{a})$, there are configurations $\beta=\left(\frac{\alpha}{\bar{i}_{1}}, \ldots, \bar{i}_{p}\right)$ and $\gamma=\left(\overline{\overline{i_{1}}}, \ldots, \overline{\overline{i_{q}}}\right)$ with $p \geq m, q \geq m, u \in F_{\beta}, v \in F_{\gamma}$. Moreover, there exist $p_{1}<p_{2}<\cdots<p_{m}=p$ and $q_{1}<q_{2}<\cdots<q_{m}=q$ such that $\overline{i_{p_{j}}}=\overline{\overline{i_{q_{j}}}}=i_{j}$ for all $j=1, \ldots, m$, and if $x_{r}(u), r \leq p$ (resp. $x_{r}(v), r \leq q$ ) is a proper reflection point of $\gamma(u)$ (resp. $\gamma(v)$ ), then $r=p_{j}$ (resp. $r=q_{j}$ ) for some $j=1, \ldots, m$.

Define $\overline{S_{t}}(u)$ (resp. $\overline{S_{t}}(v)$ ) for $t \geq 0$ in the same way as $S_{t}(u)$ (resp. $S_{t}(v)$ ) assuming that after the $p$ th (resp. $q$ th) reflection from $\partial K$ the point $u$ (resp. $v$ ) is moving straightforward with constant velocity $J_{\alpha}(u)=J_{\beta}(u)$ (resp. $\left.J_{\alpha}(v)=J_{\gamma}(v)\right)$ no matter whether it intersects $K$ or not.

Set $x_{j}=x_{p_{j}}(u)$ and $y_{j}=y_{q_{j}}(v)$ for $j=1, \ldots, m$ and take

$$
t>\max \left(t_{p}(u), t_{q}(v)\right)
$$

sufficiently large (this will be made clear afterwards). Denote by $A_{j}$ the tangent plane to $\partial K_{i_{j}}$ at $x_{j}$, by $H_{j}$ the closed half-space determined by $A_{j}$ and 
containing $K_{i_{j}}$, by $\sigma_{j}$ the symmetry map with respect to $A_{j}$, and by $\delta_{j}$ the symmetry map with respect to the tangent plane to $\partial K_{i_{j}}$ at $y_{j}$.

Further, denote by $x_{1}^{(t)}$ (resp. $\left.y_{1}^{(t)}\right)$ the point for which we have $x_{1} \in\left[u, x_{1}^{(t)}\right]$ and $\left\|u-x_{1}^{(t)}\right\|=t$ (resp. $y_{1} \in\left[v, y_{1}^{(t)}\right]$ and $\left\|v-y_{1}^{(t)}\right\|=t$ ). Let $A_{1}^{(t)}$ be the plane parallel to $Z_{\omega}$ and passing through $x_{1}^{(t)}$ and $y_{1}^{(t)}$. Set $x_{2}^{(t)}=\sigma_{1}\left(x_{1}^{(t)}\right)$, $y_{2}^{(t)}=\delta_{1}\left(y_{1}^{(t)}\right), A_{2}^{(t)}=\sigma_{1}\left(A_{1}^{(t)}\right)$, and $H_{2}^{(t)}=\sigma_{1}\left(H_{1}^{(t)}\right)$ (see Figure 1).

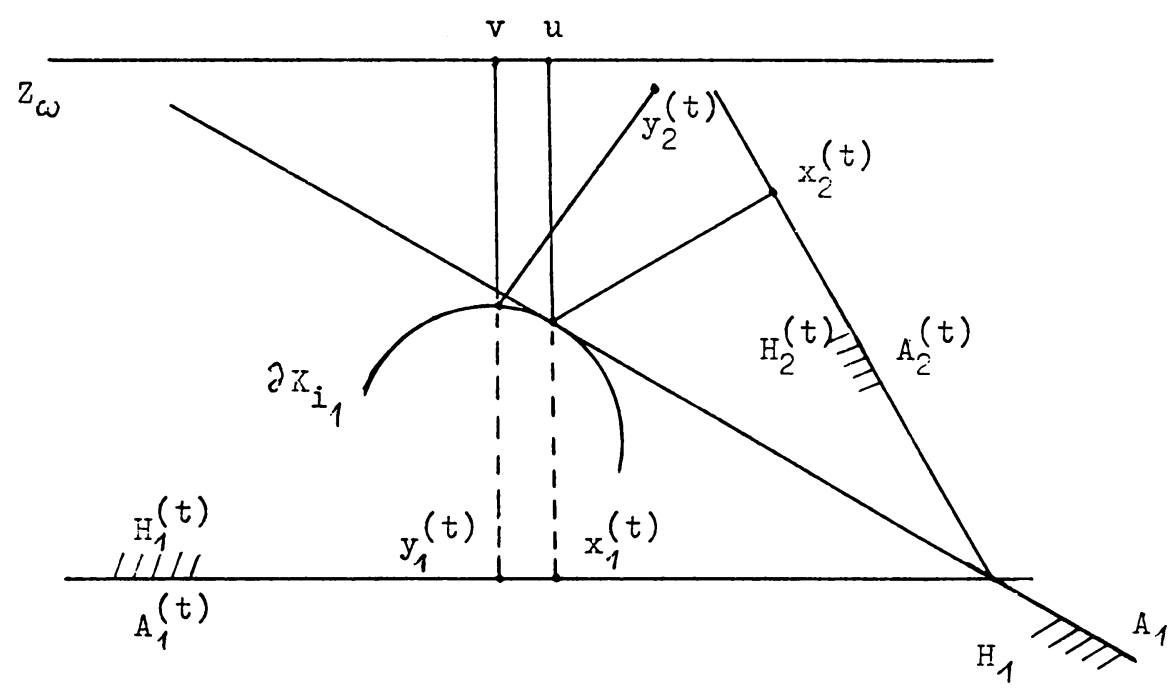

FIGURE 1

Choose $t$ so large that $K_{i_{1}} \subset \stackrel{\circ}{H}_{1}^{(t)}$. It is then clear that $K_{i_{1}} \subset \stackrel{\circ}{H}_{2}^{(t)}$. Note that if $\gamma(u)$ is tangent to $\partial K_{i_{1}}$ at $x_{1}$, then $x_{2}^{(t)}=x_{1}^{(t)}, A_{2}^{(t)}=A_{1}^{(t)}$, and $H_{2}^{(t)}=H_{1}^{(t)}$. In particular, we have

$$
y_{1} \in \stackrel{\circ}{H}_{2}^{(t)} .
$$

On the other hand, $y_{1} \in K_{i_{1}} \backslash\{x\}$ implies $y_{1} \in \stackrel{\circ}{H}_{1}$. By definition $A_{2}^{(t)}=$ $\sigma_{1}\left(A_{1}^{(t)}\right)$, therefore $\rho\left(y_{1}, A_{1}^{(t)}\right) \leq \rho\left(y_{1}, A_{2}^{(t)}\right)$ and the equality holds if and only if $\gamma(u)$ is tangent to $\partial K_{i_{1}}$ at $x_{1}$. Here $\rho(z, A)$ is the Euclidean distance between $z$ and $A$. Since $\left\|y_{1}-y_{2}^{(t)}\right\|=\left\|y_{1}-y_{1}^{(t)}\right\|=\rho\left(y_{1}, A_{1}^{(t)}\right)$, we get

$$
\left\|y_{1}-y_{2}^{(t)}\right\| \leq \rho\left(y_{1}, A_{2}^{(t)}\right),
$$

and (3.4) yields

$$
y_{2}^{(t)} \in H_{2}^{(t)} .
$$

Moreover, if $x_{1}$ or $y_{1}$ is a proper reflection point of $\gamma(u)$ or $\gamma(v)$, respectively, then $y_{2}^{(t)} \in \stackrel{\circ}{H}_{2}^{(t)}$.

Further, we proceed by induction. In fact we will describe in detail only the next step. 
Set $A_{3}^{(t)}=\sigma_{2}\left(A_{2}^{(t)}\right), \quad H_{3}^{(t)}=\sigma_{2}\left(H_{2}^{(t)}\right), x_{3}^{(t)}=\sigma_{2}\left(x_{2}^{(t)}\right)$, and $y_{3}^{(t)}=\delta_{2}\left(y_{2}^{(t)}\right)$ (see Figure 2). Since $y_{2} \in\left[y_{1}, y_{2}^{(t)}\right]$, by (3.4) and (3.6), we get $y_{2} \in \stackrel{\circ}{H}_{2}^{(t)}$. On the other hand, $y_{2} \in \stackrel{\circ}{H}_{2}$, hence $\rho\left(y_{2}, A_{2}^{(t)}\right) \leq \rho\left(y_{2}, A_{3}^{(t)}\right)$. By (3.5) we find $\left\|y_{2}-y_{2}^{(t)}\right\| \leq \rho\left(y_{2}, A_{2}^{(t)}\right)$, so

$$
\left\|y_{2}-y_{3}^{(t)}\right\| \leq \rho\left(y_{2}, A_{3}^{(t)}\right)
$$

Since $x_{2}, x_{2}^{(t)} \in H_{2} \cap H_{2}^{(t)}$, exploiting the fact that $\left[x_{2}, x_{2}^{(t)}\right]$ is orthogonal to $A_{2}^{(t)}$, we see that $y_{2} \in H_{3}^{(t)}$. Now (3.7) yields $y_{3}^{(t)} \in H_{3}^{(t)}$. Moreover, if at least one of the points $x_{1}, x_{2}, y_{1}, y_{2}$ is a proper reflection point, then $y_{3}^{(t)} \in \stackrel{\circ}{H}_{3}^{(t)}$.

Since $u \in M_{\alpha}$ means that some $x_{j}$ is a proper reflection point of $\gamma(u)$, proceeding in this way we conclude finally that

$$
y_{m+1}^{(t)} \in \stackrel{\circ}{H}_{m+1}^{(t)},
$$

where $H_{m+1}^{(t)}$ is the half-space determined by $A_{m+1}^{(t)}$ and containing $x_{m}$, while $A_{m+1}^{(t)}$ is the plane passing through $x_{m+1}^{(t)}$ and orthogonal to $\left[x_{m}, x_{m+1}^{(t)}\right]$. Let us mention that by our construction we have $x_{m+1}^{(t)}=\overline{S_{t}}(u)$ and $y_{m+1}^{(t)}=\overline{S_{t}}(v)$. Thus, by (3.8), $S_{t}(v) \in H_{m+1}^{(t)}$.

Replacing the roles of $u$ and $v$ and using the same arguments, we see that $\overline{S_{t}}(u) \in \stackrel{\circ}{G}_{m+1}^{(t)}$. Here $G_{m+1}^{(t)}$ is the half-space determined by $B_{m+1}^{(t)}$ and containing $y_{m}$, while $B_{m+1}^{(t)}$ is the plane orthogonal to $\left[y_{m}, y_{m+1}^{(t)}\right]$ and passing through $\overline{S_{t}}(v)=y_{m+1}^{(t)}$.

Suppose $J_{\alpha}(u)=J_{\alpha}(v)$. Choose $t$ so large that $K_{i_{m}} \subset \stackrel{\circ}{H}_{m+1}^{(t)} \cap \stackrel{\circ}{G}_{m+1}^{(t)}$. Then $A_{m+1}^{(t)}$ and $B_{m+1}^{(t)}$ are parallel, so either $G_{m+1}^{(t)} \subset H_{m+1}^{(t)}$ or $H_{m+1}^{(t)} \subset G_{m+1}^{(t)}$.

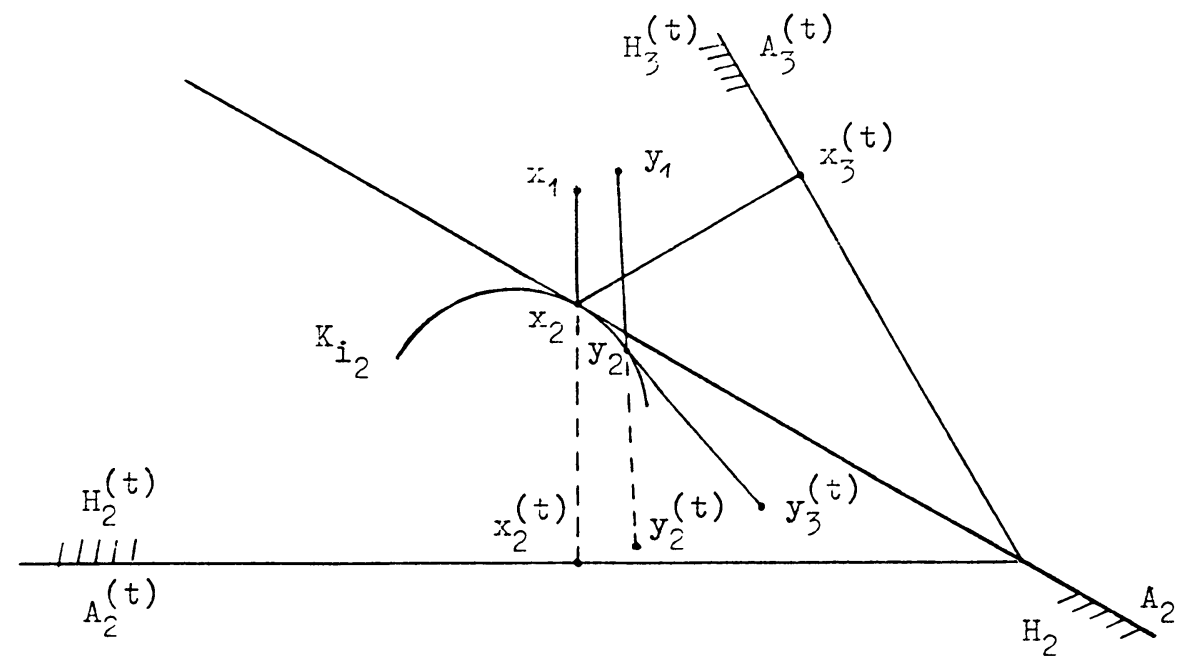

FIGURE 2 
Assume, for example, $G_{m+1}^{(t)} \subset H_{m+1}^{(t)}$. Then $\overline{S_{t}}(u) \in G_{m+1}^{(t)} \subset H_{m+1}^{(t)}$, hence $\overline{S_{t}}(u) \notin A_{m+1}^{(t)}$, which gives a contradiction. Therefore $J_{\alpha}(u) \neq J_{\alpha}(v)$ and the proof of the theorem is complete.

Let $\omega, \theta \in S^{n-1}$ and let $\gamma$ be an $(\omega, \theta)$-ray with successive reflection points $x_{1}, \ldots, x_{m}$. We say that $\gamma$ is of type $\alpha=\left(i_{1}, \ldots, i_{m}\right)$ if $x_{j} \in \partial K_{i_{j}}$ for every $j=1, \ldots, m$.

Corollary 3.3. If $\omega \neq \theta$, then for every configuration $\alpha$ there exists at most one $(\omega, \theta)$-ray of type $\alpha$.

It is clear that in general there could exist some configurations $\alpha$ for which there are no $(\omega, \theta)$-rays of type $\alpha$. The existence of $(\omega, \theta)$-rays will be treated in $\S 6$.

Fix a configuration $\alpha=\left(i_{1}, \ldots, i_{m}\right)$ and consider the map $J_{\alpha}: U_{\alpha} \rightarrow S^{n-1}$. For our aims in $\S 5$ we will show that $d J_{\alpha}(u)$ is invertible for each $u \in U_{\alpha}$. The proof of this fact is based on the representation of the Poincare map related to a periodic billiard trajectory found by Petkov and Vogel [18] (see also $\S \S 4,5$ in [21]).

Let $u_{0} \in U_{\alpha}$ and let $Q_{j}=x_{j}\left(u_{0}\right), j=1, \ldots, m$. For $j=1, \ldots, m-1$ denote by $D_{j}$ the oriented line $Q_{j} Q_{j+1}$ with direction $\vec{Q}_{j} Q_{j+1}$. By $D_{m}$ we denote the oriented line through $Q_{m}$ with direction $\theta=J_{\alpha}\left(u_{0}\right)$. Choose a point $X_{j} \in D_{j}$ so that $\left\|Q_{j} X_{j}\right\|=1$ and ${\overrightarrow{Q_{j}}}_{j}$ is colinear with ${\overrightarrow{Q_{j} Q_{j+1}}}_{\text {for }}$ $j<m$ and ${\overrightarrow{Q_{m} X}}_{m}=\theta$. By $\Pi_{j}$ we denote the hyperplane passing through $X_{j}$ and orthogonal to $D_{j}$. Let $\sigma_{j}$ be the symmetry map with respect to the tangent plane $A_{j}$ to $\partial K$ at $Q_{j}$ and let $\lambda_{j}=\left\|Q_{j} Q_{j-1}\right\|$ for $j=2, \ldots, m$, $\lambda_{1}=\left\|u_{0} Q_{1}\right\|+1$. Then

$$
Z_{\omega} \times Z_{\omega} \ni(u, v) \rightarrow\left(u+\lambda_{1} v, v\right) \in Z_{1} \times Z_{1},
$$

where we identify $Z_{\omega}$ and $Z_{1}=\sigma_{1}\left(\Pi_{1}\right)$ by the orthogonal projection of $Z_{\omega}$ onto $Z_{1}$. Let $\pi_{j}: \Pi_{j-1} \rightarrow T_{Q_{j}}(\partial K)$ be the projection along the direction $\vec{Q}_{j-1} Q_{j}, \quad j=2, \ldots, m$, and let $G_{j}$ be the differential of the Gauss map corresponding to $\partial K$ at $Q_{j}$. Consider the symmetric linear map $\widetilde{\psi}_{j}: \Pi_{j} \rightarrow \Pi_{j}$, given by

$$
\left\langle\widetilde{\psi}_{j} \sigma_{j} w, \sigma_{j} w\right\rangle=-2\left\langle{\overrightarrow{Q_{j-1} Q_{j}}}, \nu\left(Q_{j}\right)\right\rangle\left\langle G_{j} \pi_{j}(w), \pi_{j}(w)\right\rangle /\left\|Q_{j-1} Q_{j}\right\|
$$

for $w \in \Pi_{j-1}$ (we identify here and below the planes $\sigma_{j}\left(\Pi_{j-1}\right)$ and $\Pi_{j}$ according to the orthogonal projection of one of them onto the other). Following the argument and the basis given in $[18,21]$, we have

$$
\left(d J_{\alpha}\left(u_{0}\right)\right)(u)=p r_{2}\left(\begin{array}{cc}
\sigma_{m} & \lambda_{m} \sigma_{m} \\
\widetilde{\psi}_{m} \sigma_{m} & \sigma_{m}+\lambda_{m} \widetilde{\psi}_{m} \sigma_{m}
\end{array}\right) \cdots\left(\begin{array}{cc}
\sigma_{1} & \lambda_{1} \sigma_{1} \\
\widetilde{\psi}_{1} \sigma_{1} & \sigma_{1}+\lambda_{1} \widetilde{\psi}_{1} \sigma_{1}
\end{array}\right)\left(\begin{array}{c}
u \\
0
\end{array}\right)
$$

for $u \in Z_{\omega}$, where $p r_{2}(u, v)=v$. 
Proposition 3.4. For every configuration $\alpha$ we have

$$
\begin{array}{r}
\left(d J_{\alpha}\left(u_{0}\right)\right)(u)=M_{m} \sigma_{m}\left(I_{m-1}+\lambda_{m} M_{m-1}\right) \sigma_{m-1}\left(I_{m-2}+\lambda_{m-1} M_{m-2}\right) \\
\cdots \\
\cdots \sigma_{2}\left(I_{1}+\lambda_{2} M_{1}\right) \sigma_{1} u,
\end{array}
$$

where $I_{i}$ is the identity map on $\Pi_{i}$ and for $i=1, \ldots, m, M_{i}: \Pi_{i} \rightarrow \Pi_{i}$ is a symmetric positive definite linear map. Moreover,

(3.11) $M_{1}=\widetilde{\psi}_{1}$,

$$
M_{i}=\sigma_{i} M_{i-1}\left(I_{i-1}+\lambda_{i} M_{i-1}\right)^{-1} \sigma_{i}+\widetilde{\psi}_{i}
$$$$
i=2, \ldots, m \text {. }
$$

The proof is straightforward and we omit it. Consequently, we get

Corollary 3.5. For every configuration $\alpha$ and every $u_{0} \in U_{\alpha}$ the map $d J_{\alpha}\left(u_{0}\right)$ is invertible and $J_{\alpha}: U_{\alpha} \rightarrow J_{\alpha}\left(U_{\alpha}\right)$ is a diffeomorphism.

\section{4. $(\omega, \theta)$-RAYS FOR GENERIC $\theta$}

In this section we prove that for given $\omega \in S^{n-1}$ there exists a residual subset $\mathscr{R}=\mathscr{R}(\omega)$ of $S^{n-1}$ such that for $\theta \in \mathscr{R}$ all $(\omega, \theta)$-rays have some special properties. Recall that a subset $\mathscr{R}$ of a topological space $X$ is called residual in $X$ if $\mathscr{R}=\bigcap_{i=1}^{\infty} U_{i}$, where $U_{i}$ are open and dense subsets of $X$.

Throughout this section $\omega \in S^{n-1}$ will be fixed.

Theorem 4.1. There exists a residual subset $\mathscr{R}_{1}=\mathscr{R}_{1}(\omega)$ of $S^{n-1}$ so that for every $\theta \in \mathscr{R}_{1}$ and every configuration $\alpha$ if $u \in F_{\alpha}$ and $J_{\alpha}(u)=\theta$, then $u \in U_{\alpha}$.

The latter means that if $J_{\alpha}(u) \in \mathscr{R}_{1}$ for some $u \in F_{\alpha}$, then the first $|\alpha|$ reflection points of $\gamma(u)$ are proper ones.

Proof. We are going to construct a sequence $\mathscr{T}_{1} \supset \mathscr{T}_{2} \supset \cdots \supset \mathscr{T}_{m} \supset \cdots$ of open and dense subsets of $S^{n-1}$ so that for every $m$ if $\alpha$ is a configuration with $|\alpha|=m, u \in F_{\alpha}$ and $J_{\alpha}(u) \in \mathscr{T}_{m}$, then the first $m$ reflection points of $\gamma(u)$ are proper ones.

Set $\mathscr{T}_{1}=S^{n-1} \backslash\{\omega\}$. Suppose we have constructed open and dense subsets $\mathscr{T}_{1} \supset \cdots \supset \mathscr{T}_{m}$ of $S^{n-1}$ having the desired properties.

Fix a configuration $\alpha=\left(i_{1}, \ldots, i_{m}, i_{m+1}\right)$ and set $\alpha_{k}=\left(i_{1}, \ldots, i_{k}\right)$ for $k=1, \ldots, m$. Since $\mathscr{T}_{m}$ is open and dense, $F=S^{n-1} \backslash \mathscr{T}_{m}$ is compact and $\stackrel{\circ}{F}=\varnothing$. Let $\beta=\alpha_{k}$ for some $k=1, \ldots, m$. Then $F \cap E_{\beta}$ is compact with empty interior (cf. (3.3)). Consider the map $J_{\beta}: \overline{F_{\beta}} \rightarrow E_{\beta} \subset S^{n-1}$. Since

$$
J_{\beta}^{-1}(F)=J_{\beta}^{-1}\left(F \cap E_{\beta}\right) \subset\left(J_{\beta}^{-1}\left(F \cap E_{\beta}\right) \cap M_{\alpha}\right) \cup L_{\alpha},
$$

we deduce from Theorem 3.2 that $J_{\beta}^{-1}(F)$ has empty interior in $Z_{\omega}$. Hence $F_{\alpha} \cap J_{\beta}^{-1}(F)$ is a compact subset of $Z_{\omega}$ with empty interior. Thus $J_{\alpha}\left(F_{\alpha} \cap J_{\beta}^{-1}(F)\right)$ is compact, and as is easily seen from Theorem 3.2, it has empty interior in $S^{n-1}$. In this way we have proved that

$$
V=\mathscr{T}_{m} \backslash \bigcup_{k=1}^{m} J_{\alpha}\left(F_{\alpha} \cap J_{\alpha_{k}}^{-1}(F)\right)
$$




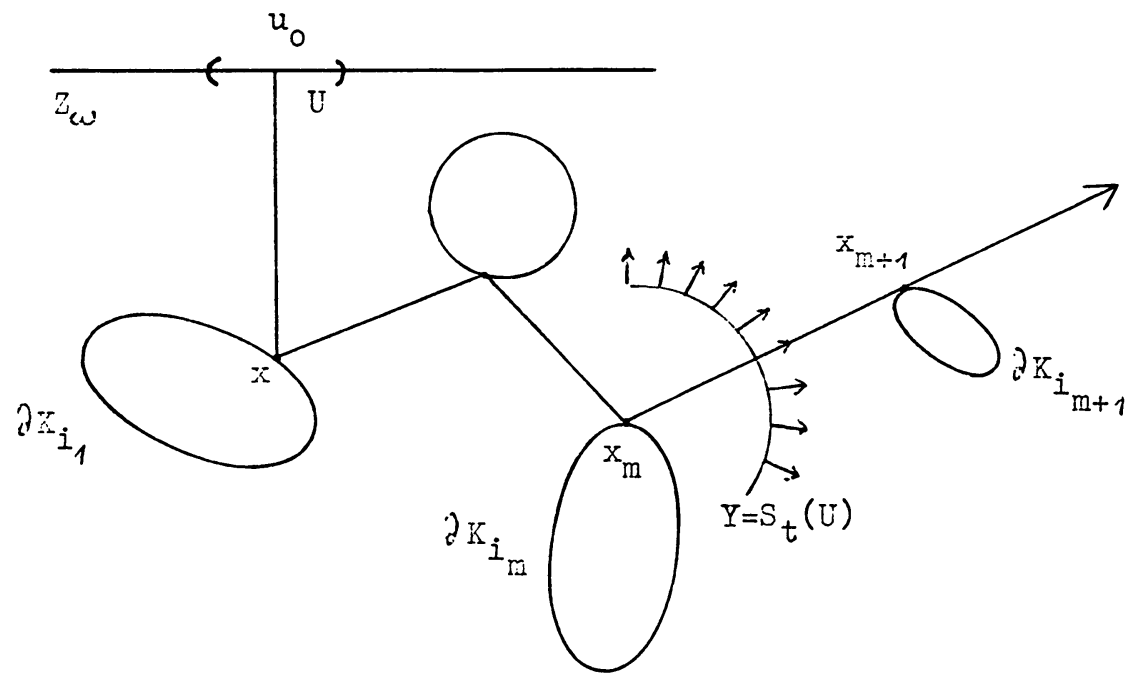

FIGURE 3

is an open and dense subset of $S^{n-1}$. Note that if $u \in F_{\alpha}$ and $J_{\alpha}(u) \in V$, then the first $m$ reflection points of $\gamma(u)$ are proper ones.

Denote by $\mathscr{T}_{m+1}^{(\alpha)}$ the set of $\theta \in V$ such that if $J_{\alpha}(u) \in \theta$ for some $u \in F_{\alpha}$, then the first $m+1$ reflection points of $\gamma(u)$ are proper ones. We are going to show that $\mathscr{T}_{m+1}^{(\alpha)}$ is open and dense in $V$ (and therefore in $S^{n-1}$ ). Clearly, $\mathscr{T}_{m+1}^{(\alpha)}$ is open. To prove the density, take $\theta \in V \backslash \mathscr{T}_{m+1}^{(\alpha)}$. Then $\theta=J_{\alpha}\left(u_{0}\right)$ for some $u_{0} \in F_{\alpha}$, the first $m$ reflection points $x_{1}\left(u_{0}\right), \ldots, x_{m}\left(u_{0}\right)$ of $\gamma\left(u_{0}\right)$ are proper ones, and $\gamma\left(u_{0}\right)$ is tangent to $\partial K$ at the $(m+1)$ st reflection point $x_{m+1}\left(u_{0}\right)$. Choose $t$ so that $t_{m}\left(u_{0}\right)<t<t_{m+1}\left(u_{0}\right)$ and take a neighborhood $U$ of $u_{0}$ in $Z_{\omega}$ so small that $U \subset \stackrel{\circ}{F}_{\alpha}=U_{\alpha}$. By Proposition 2.1 it follows that for $U$ sufficiently small $Y=S_{t}(U)$ is a smooth strictly convex surface in $\mathbf{R}^{n}$ (see Figure 3). Then for every $u \in U$ the ray $\gamma(u)$ issued from $u$ has exactly $m$ proper reflections from $\partial K_{i_{1}}, \ldots, \partial K_{i_{m}}$ when the time runs from 0 to $t$. Note that $\left\{N_{t}(u): u \in U\right\}$ is a normal field for $Y$ and if $u \in U$, then $S_{t}(u)$ moves in the direction $N_{t}(u)$ (cf. subsection 2.2). It is now clear that there exists $u^{\prime} \in U$ such that the ray starting at $S_{t}\left(u^{\prime}\right) \in Y$ with direction $N_{t}\left(u^{\prime}\right)$ intersects transversely $\partial K_{i_{m+1}}$. This means that $J_{\alpha}\left(u^{\prime}\right) \in \mathscr{T}_{m+1}^{(\alpha)}$. We have shown in this way that $\mathscr{T}_{m+1}^{(\alpha)}$ is open and dense in $S^{n-1}$.

Setting $\mathscr{T}_{m+1}=\bigcap_{|\alpha|=m+1} \mathscr{T}_{m+1}^{(\alpha)}$, we get an open and dense subset of $S^{n-1}$ with the desired properties. This closes the induction.

Finally, setting $\mathscr{R}_{1}=\bigcap_{m=1}^{\infty} \mathscr{T}_{m}$, we complete the proof of the theorem.

Let $B_{a}$ be an open ball with radius $a>0$ containing $K$ such that $Z_{\omega}$ is tangent to $\overline{B_{a}}$. For $\theta \in S^{n-1}$ denote by $Z_{\theta}$ the tangent plane to $\overline{B_{a}}$ such that $\theta$ is orthogonal to $Z_{\theta}$ and the half-space $H_{\theta}$ determined by $Z_{\theta}$ and having 
$\theta$ as an outward normal contains $B_{a}$. Let $\gamma$ be an $(\omega, \theta)$-ray with successive reflection points $x_{1}, \ldots, x_{m}$. The sojourn time $T_{\gamma}$ of $\gamma$ is defined by

$$
T_{\gamma}=\left\|\pi_{\omega}\left(x_{1}\right)-x_{1}\right\|+\sum_{i=1}^{m-1}\left\|x_{i}-x_{i+1}\right\|+\left\|x_{m}-\pi_{\theta}\left(x_{m}\right)\right\|-2 a,
$$

where $\pi_{\omega}: \mathbf{R}^{n} \rightarrow Z_{\omega}$ and $\pi_{\theta}: \mathbf{R}^{n} \rightarrow Z_{\theta}$ are the orthogonal projections. The definition of $T_{\gamma}$ was introduced by Guillemin [5]. It is easy to see that $T_{\gamma}$ does not depend on $a$.

Theorem 4.2. There exists a residual subset $\mathscr{R}_{2}=\mathscr{R}_{2}(\omega)$ of $S^{n-1}$ such that if $\theta \in \mathscr{R}_{2}$, then for any two different $(\omega, \theta)$-rays $\gamma$ and $\delta$ we have $T_{\gamma} \neq T_{\delta}$.

Proof. First, notice that if $\gamma$ is an $(\omega, \theta)$-ray, then there exist $u \in Z_{\omega}$ and $m \in \mathbf{N}$ so that $x_{1}(u), \ldots, x_{m}(u)$ are the successive reflection points of $\gamma$. Moreover, there is unique $t>t_{m}(u)$ with $S_{t}(u) \in Z_{\theta}$ and then we have $N_{t}(u)=$ $\theta$ and $T_{\gamma}=t-2 a$.

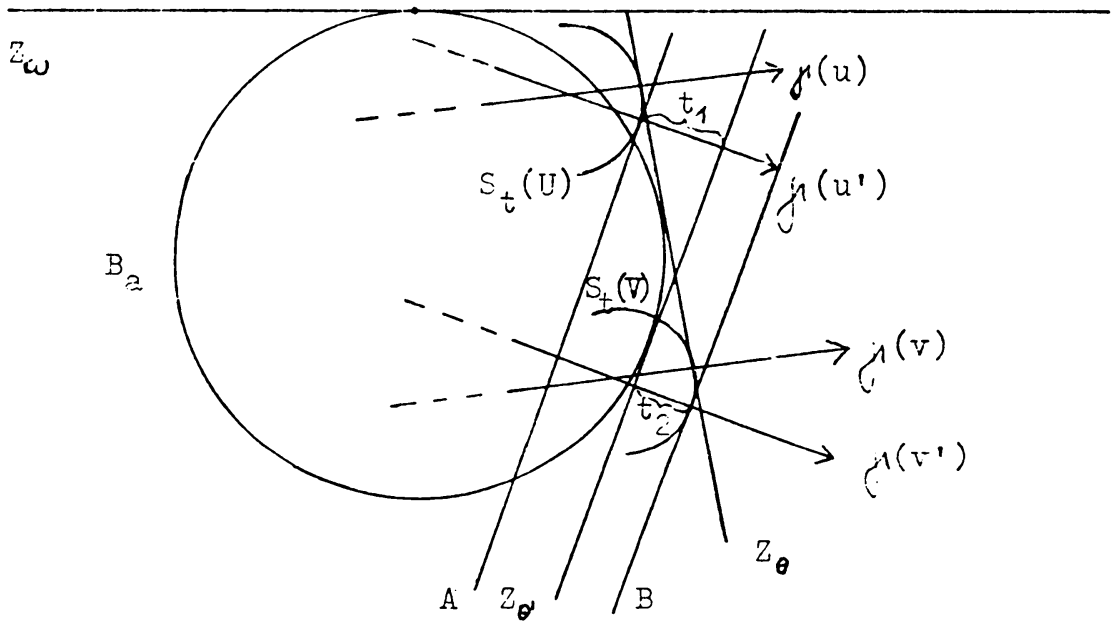

Figure 4

Let $u \in Z_{\omega}$ be such that $1 \leq r(u)<\infty$. Setting $m_{1}=r(u)$ and $\theta=N_{t}(u)$ for $t>t_{m_{1}}(u)$, choose uniquely $t_{0}$ with $S_{t_{0}}(u) \in Z_{\theta}$ and set $T_{\theta}(u)=t_{0}$.

Further, fix two different configurations $\alpha$ and $\beta$ and set $m=\max (|\alpha|,|\beta|)$ and

$$
\begin{aligned}
& \mathscr{L}(\alpha, \beta)=\left\{\theta \in \mathscr{T}_{m}: \text { if } u \in U_{\alpha}, v \in U_{\beta}, r(u)=|\alpha|, r(v)=|\beta|,\right. \\
& \text { and } \left.J_{\alpha}(u)=J_{\beta}(v)=\theta, \text { then } T_{\theta}(u) \neq T_{\theta}(v)\right\} .
\end{aligned}
$$

Here $\mathscr{T}_{m}$ is the set defined in the proof of Theorem 4.1. We are going to prove $\mathscr{L}(\alpha, \beta)$ is open and dense in $\mathscr{T}_{m}$.

To show $\mathscr{T}_{m} \backslash \mathscr{L}(\alpha, \beta)$ is closed in $\mathscr{T}_{m}$, consider a sequence $\left\{\theta_{k}\right\} \subset$ $\mathscr{G}_{m} \backslash \mathscr{L}(\alpha, \beta)$ with $\theta_{k} \rightarrow \theta \in \mathscr{T}_{m}$. Then for any $k$ there exist $u_{k} \in U_{\alpha}$ 
and $v_{k} \in U_{\beta}$ with $r\left(u_{k}\right)=|\alpha|, r\left(v_{k}\right)=|\beta|, J_{\alpha}\left(u_{k}\right)=J_{\beta}\left(v_{k}\right)=\theta_{k}$, and $T_{\theta_{k}}\left(u_{k}\right)=T_{\theta_{k}}\left(v_{k}\right)$. We may assume $u_{k} \rightarrow u \in \overline{F_{\alpha}}$ and $v_{k} \rightarrow v \in \overline{F_{\beta}}$. If $u \notin F_{\alpha}$, then $u \in F_{\alpha^{\prime}}$ for some $\alpha^{\prime}$ and $\gamma(u)$ would have some tangent point to $\partial K$. On the other hand,

$$
J_{\alpha^{\prime}}(u)=J_{\alpha}(u)=\lim _{k \rightarrow \infty} J_{\alpha}\left(u_{k}\right)=\lim _{k \rightarrow \infty} \theta_{k}=\theta \in \mathscr{T}_{m},
$$

which is a contradiction with the properties of $\mathscr{T}_{m}$. So $u \in F_{\alpha}$ and again by $J_{\alpha}(u)=\theta \in \mathscr{T}_{m}$ we get $u \in U_{\alpha}$. Similarly, $J_{\beta}(v)=\theta$ and $v \in U_{\beta}$. It is clear also that $r(u)=|\alpha|$ and $r(v)=|\beta|$. Therefore $\theta \in \mathscr{T}_{m} \mid \mathscr{L}(\alpha, \beta)$ and we have shown that $\mathscr{L}(\alpha, \beta)$ is open in $\mathscr{T}_{m}$.

To establish the density, take $\theta \in \mathscr{T}_{m}$ and suppose $\theta \notin \mathscr{L}(\alpha, \beta)$. Then there exist $u \in U_{\alpha}, v \in U_{\beta}$ with $r(u)=|\alpha|, r(v)=|\beta|, J_{\alpha}(u)=J_{\beta}(v)=\theta$, and $T_{\theta}(u)=T_{\theta}(v)$. It follows by Proposition 2.1 that for $t=T_{\theta}(u)=T_{\theta}(v)$ there exist small open neighborhoods $U \subset U_{\alpha}$ and $V \subset U_{\beta}$ of $u$ and $v$, respectively, in $Z_{\omega}$ such that $S_{t}(U)$ and $S_{t}(V)$ are strictly convex surfaces. Note that both $S_{t}(U)$ and $S_{t}(V)$ are tangent to $Z_{\theta}$ at $S_{t}(u)$ and $S_{t}(v)$, respectively, hence they lie in $H_{\theta}$ (see Figure 4). Further, according to Theorem 3.2 , we see that for every $\theta^{\prime} \in \mathscr{T}_{m}$ sufficiently close to $\theta$ there exist unique $u^{\prime} \in U$ and $v^{\prime} \in V$ with $J_{\alpha}\left(u^{\prime}\right)=J_{\beta}\left(v^{\prime}\right)=\theta^{\prime}$. Denote by $F$ the set of those $\theta^{\prime} \in \mathscr{T}_{m}$ with $J_{\alpha}\left(u^{\prime}\right)=J_{\beta}\left(v^{\prime}\right)=\theta^{\prime}$ for some $u^{\prime} \in U$ and $v^{\prime} \in V$ and such that there exists a plane tangent simultaneously to $S_{t}(U)$ at $S_{t}\left(u^{\prime}\right)$ and to $S_{t}(V)$ at $S_{t}\left(v^{\prime}\right)$. It is easy to see that $\stackrel{\circ}{F}=\varnothing$. Since $\mathscr{T}_{m}$ is open in $S^{n-1}$, we can take $\theta^{\prime} \in \mathscr{T}_{m} \backslash F$ arbitrarily close to $\theta$. Choosing such a $\theta^{\prime}$, denote by $A$ (resp. $B$ ) the plane tangent to $S_{t}(U)$ (resp. $S_{t}(V)$ ) at $S_{t}\left(u^{\prime}\right)$ (resp. $S_{t}\left(v^{\prime}\right)$ ). Then the three planes $Z_{\theta^{\prime}}, A$, and $B$ are mutually parallel. Thus for $t_{1}=T_{\theta^{\prime}}\left(u^{\prime}\right)-t$ and $t_{2}=T_{\theta^{\prime}}\left(v^{\prime}\right)-t$, we see that $\left|t_{1}\right|$ is the distance between $A$ and $Z_{\theta^{\prime}}$, while $\left|t_{2}\right|$ is the distance between $B$ and $Z_{\theta^{\prime}}$. Since $A \neq B$, we have $t_{1} \neq t_{2}$, hence $T_{\theta^{\prime}}\left(u^{\prime}\right)=t+t_{1} \neq t+t_{2}=T_{\theta^{\prime}}\left(v^{\prime}\right)$. On the other hand, $J_{\alpha}$ is invertible on $U_{\alpha}$, while $J_{\beta}$ is invertible on $U_{\beta}$. Consequently, $\theta^{\prime} \in \mathscr{L}(\alpha, \beta)$ and $\mathscr{L}(\alpha, \beta)$ is dense in $\mathscr{T}_{m}$.

Setting

$$
\mathscr{R}_{2}=\bigcap_{\alpha \neq \beta} \mathscr{L}(\alpha, \beta),
$$

we obtain a residual subset of $S^{n-1}$ having the desired properties. This proves the theorem.

\section{SiNGULARITIES OF THE SCATTERING KERNEL}

In this section we prove Theorem 1.1. The scattering kernel $s(t, \theta, \omega)$ is given by (1.1). Let $\rho(t) \in C_{0}^{\infty}(\mathbf{R}), \rho(t)=1$ for $|t| \leq 1 / 2, \rho(t)=0$ for 
$|t| \geq 1$. Setting

$$
\rho_{\varepsilon}(t)=\rho(t / \varepsilon), \quad 0<\varepsilon \leq 1, \quad \rho_{\varepsilon}^{(k)}=\frac{\partial^{k} \rho_{\varepsilon}}{\partial t^{k}}
$$

consider

$$
\begin{aligned}
& \left(s(t, \theta, \omega), \rho_{\varepsilon}\left(t+T_{0}\right) e^{-i t \lambda}\right) \\
& =\sum_{k=0}^{n-2} c_{k}(-i \lambda)^{n-2-k} \int_{\mathbf{R}} \int_{\partial K} e^{i \lambda(t-\langle x, \theta\rangle)} \cdot \rho_{\varepsilon}^{(k)}\left(\langle x, \theta\rangle-t+T_{0}\right) \\
& \times \frac{\partial w}{\partial \nu}(t, x, \omega) d t d S_{x},
\end{aligned}
$$

where $c_{k}=$ const, $c_{0}=C_{n}$. Obviously, we must study $\partial w /\left.\partial \nu\right|_{\mathbf{R} \times \partial K}$ only for $(t, x)$ satisfying the relation

$$
\begin{aligned}
\mathrm{WF}\left(\left.\frac{\partial w}{\partial \nu}\right|_{\mathbf{R} \times \partial K}\right) & \cap\left\{\left(t, x, 1,-\left.\operatorname{grad}\langle x, \theta\rangle\right|_{\partial K}\right) \in T^{*}(\mathbf{R} \times \partial K)\right\} \\
& \cap\left\{(t, x, 1, \xi):(t, x) \in \operatorname{supp} \rho_{\varepsilon}^{(k)}\left(\langle x, \theta\rangle-t+T_{0}\right)\right\} \neq \varnothing .
\end{aligned}
$$

It is well known [14] that the generalized wave front $\operatorname{WF}_{b}(w)$ is propagating along the outgoing generalized bicharacteristics of the operator $\square$ issued from $\mathrm{WF}\left(\delta\left(-\rho_{0}-\langle x, \omega\rangle\right)\right)$. The projections of these bicharacteristics on $\bar{\Omega}$ will be called generalized rays. They are unions of finite or infinite number segments which are reflecting or tangent to $\partial K$.

The results about the propagation of singularities [14] show that $(t, x, 1, \eta) \in$ $\mathrm{WF}\left(\partial w /\left.\partial \nu\right|_{\mathbf{R} \times \partial K}\right)$ if there exists $\tilde{\eta} \in T_{x}^{*}(\bar{\Omega})$ such that $|\tilde{\eta}|=1,\left.\tilde{\eta}\right|_{T_{x}(\partial K)}=\eta$, $\langle\tilde{\eta}, \nu(x)\rangle \leq 0$. Therefore $\left.\tilde{\eta}\right|_{T_{x}(\partial K)}=-\left.\theta\right|_{T_{x}(\partial K)}$ leads to one of the following cases:

(a) $\tilde{\eta}=-\theta$ for $\langle\theta, \nu(x)\rangle>0$,

(b) $\tilde{\eta}=-\theta+2\langle\theta, \nu(x)\rangle \nu(x)$ for $\langle\theta, \nu(x)\rangle<0$,

(c) $\tilde{\eta}=-\theta$ for $\langle\theta, \nu(x)\rangle=0$.

In cases (a) and (b) the singularities are propagating along a generalized ray $\gamma$ reflecting at $x$. Moreover, in case (a) (resp. (b)) the reflecting (resp. the incoming) direction of $\gamma$ at $x$ is just $\theta$. In case (c) the singularities are propagating along a ray simply tangent at $x$ and having direction $\theta$. Thus we must study the behavior of the generalized rays having at least one (finite or infinite) segment with direction $\theta$.

Fix $T>0$. We are going to study the singularities $T_{0},\left|T_{0}\right| \leq T$, of $s(t, \theta, \omega)$ introducing a partition of unity $\sum_{j=1}^{\infty} \varphi_{j}\left(x^{\prime}\right)=1, \varphi_{j}\left(x^{\prime}\right)$ $\in C_{0}^{\infty}\left(\mathbf{R}^{n-1}\right), x^{\prime}=\left(x_{1}, \ldots, x_{n-1}\right)$, depending on $T$. From

$$
x \in \partial K, \quad(t, x) \in \operatorname{supp} \rho_{\varepsilon}^{(k)}\left(\langle x, \theta\rangle-t+T_{0}\right), \quad\left|T_{0}\right| \leq T
$$

we conclude that we need to know the singularities of $\partial w /\left.\partial \nu\right|_{\mathbf{R} \times \partial K}$ for $|t| \leq$ $T_{1}=T+\rho_{0}+\varepsilon$. Since the singularities of $w$ are propagating with speed 1 , 
we are going to investigate only the generalized rays $\gamma$ with lengths less than or equal to some fixed number $T_{2}$ depending on $T$ and $\rho_{0}$. Without loss of generality we may assume $\omega=(0, \ldots, 0,1)$.

Consider the Cauchy problem

$$
\left\{\begin{array}{l}
\square v_{j}=0 \\
\left.v_{j}\right|_{t=\tau}=\varphi_{j}\left(x^{\prime}\right) \delta\left(\tau-x_{n}\right) \\
\left.\frac{\partial v_{j}}{\partial t}\right|_{t=\tau}=\varphi_{j}\left(x^{\prime}\right) \delta^{\prime}\left(\tau-x_{n}\right)
\end{array}\right.
$$

with $\tau<-\rho_{0}, \tau$ fixed. It is easy to see that

$$
\begin{aligned}
& \mathrm{WF}\left(v_{j}\right) \subset\left\{(t, x, \pm 1, \mp \omega) \in T^{*}\left(\mathbf{R}^{n+1}\right) \backslash 0: \text { there exist } \hat{x} \text { and } \sigma \geq 0\right. \\
&\text { so that } \left.\hat{x}_{n}=\tau, t=\tau \pm \sigma, x=\hat{x} \pm \sigma \omega\right\} .
\end{aligned}
$$

Therefore there exists a compact set $F_{0}^{\prime} \subset \mathbf{R}^{n-1}$ such that $\operatorname{supp} \varphi_{j} \cap F_{0}^{\prime}=\varnothing$ implies singsupp $v_{j} \cap K=\varnothing$. Set

$$
F_{0}=\left\{x \in \mathbf{R}^{n}: x^{\prime} \in F_{0}^{\prime}, x_{n}=\tau\right\}
$$

and consider the generalized rays $\gamma(u)$ starting at $u \in F_{0}$ with direction $\omega$ and having lengths less than or equal to $T_{2}$. We identify $F_{0}$ with $Z_{\omega}$ and choose $\mathscr{R}(\omega)=\mathscr{R}_{1}(\omega) \cap \mathscr{R}_{2}(\omega)$, where $\mathscr{R}_{i}(\omega), i=1,2$, are defined in Theorems 4.1 and 4.2. The rays $\gamma(u)$ have at most $m_{0}$ reflections and there exists a finite number of configurations $\alpha$ with $|\alpha| \leq m_{0}$. Below we assume $\theta \in \mathscr{R}(\omega)$ fixed, $\theta \neq \omega$.

Let $\gamma(u), u \in F_{\alpha} \cap F_{0}$ be a ray having at least one segment with direction $\theta$ starting at $x_{r}(u)$. According to Theorem 4.1, the successive reflection points $x_{i}(u), \quad 1 \leq i \leq r$, of $\gamma(u)$ are proper ones and $u \in U_{\beta}$ for some $|\beta|=r$. Exploiting the continuity of the broken Hamiltonian flow related to $\square$ (see [14]) for rays with uniformly bounded lengths and Corollary 3.3 , we conclude that the points $u \in F_{0}$ with the above property of $\gamma(u)$ form a finite set $\left\{u_{1}, \ldots, u_{N}\right\}$. Thus, if $u_{0} \in F_{0} \backslash\left\{u_{1}, \ldots, u_{N}\right\}$, then the ray $\gamma\left(u_{0}\right)$ has no segments with direction $\theta$ among the first $m_{0}$ ones. Choosing a sufficienly small neighborhood $\mathscr{O}\left(u_{0}\right)$ of $u_{0}$ in $\mathbf{R}^{n-1}$, we arrange the same property for $\gamma(u)$ for all $u \in \mathscr{O}\left(u_{0}\right)$.

Given $u_{i}, i=1, \ldots, N$, there are two cases. First, assume $x_{p}\left(u_{i}\right)$ is a proper reflection point for every $p \leq m_{0}$. Taking a small neighborhood $\mathscr{O}\left(u_{i}\right)$ of $u_{i}$, we obtain that for every $u \in \mathscr{O}\left(u_{i}\right)$ the first $m_{0}$ reflection points of $\gamma(u)$ are proper ones. Second, let $\gamma\left(u_{i}\right)$ have some tangent segments among the first $m_{0}$ ones. Since $\theta \in \mathscr{R}_{1}(\omega)$, these segments have directions different from $\theta$. The continuity of the broken Hamiltonian flow implies that there exists a neighborhood $\mathscr{O}\left(u_{i}\right)$ of $u_{i}$ so that for $u \in \mathscr{O}\left(u_{i}\right)$ the ray $\gamma(u)$ has reflection points

$$
x_{1}(u), \ldots, x_{r_{0}}(u), x_{r_{0}+1}(u), \ldots, x_{q}(u), \quad r_{0} \geq 1
$$


where $x_{i}(u), i=1, \ldots, r_{0}$ are proper ones, while the segments starting at $x_{i}(u), i=r_{0}, \ldots, q$, have directions different from $\theta$. Notice that $r_{0}=r_{0}(i)$ does not depend on $u \in \mathscr{O}\left(u_{i}\right)$, while $q$ could depend on it. Finally, assume that $u_{i} \notin \mathscr{O}\left(u_{j}\right)$ for $i \neq j$.

This procedure leads to a covering $F_{0} \subset \bigcup_{u_{0} \in F_{0}} \mathscr{O}\left(u_{0}\right)$ and we may assume

$$
F_{0} \subset \bigcup_{j=1}^{M} \mathscr{O}\left(u_{j}\right), \quad N \leq M
$$

Let $\tilde{\mathscr{O}}\left(u_{j}\right) \subset \mathscr{O}\left(u_{j}\right)$ be a neighborhood of $u_{j}$. Choose the partition of unity $\left\{\varphi_{j}\left(x^{\prime}\right)\right\}_{j=1}^{\infty}$ so that

$$
\operatorname{supp} \varphi_{j} \subset \mathscr{O}\left(u_{j}\right), \varphi_{j}\left(x^{\prime}\right)=1 \text { for } x^{\prime} \in \tilde{\mathscr{O}}\left(u_{j}\right), 1 \leq j \leq M .
$$

Assume that for $M_{0} \leq N$ the rays $\gamma\left(u_{i}\right), i=1, \ldots, M_{0}$, have only proper reflection points among the first $m_{0}$ ones; that is, the first case described above holds.

Setting $V_{j}=v_{j}-\varphi_{j}\left(x^{\prime}\right) \delta\left(t-x_{n}\right), j=1, \ldots, N$, we have

$$
\left\{\begin{array}{l}
\square V_{j}=\left(\Delta_{x^{\prime}} \varphi_{j}\right) \delta\left(t-x_{n}\right) \\
\left.V_{j}\right|_{t=\tau}=\left.\frac{\partial V_{j}}{\partial t}\right|_{t=\tau}=0
\end{array}\right.
$$

and the singularities of $V_{j}$ are propagating along the straight lines $l(u)$ issued from $\mathscr{O}\left(u_{j}\right) \backslash \tilde{\mathscr{O}}\left(u_{j}\right)$ with direction $\omega$.

Fix $1 \leq j \leq N$ and consider the lines $l(u)$ with $u \in \mathscr{O}\left(u_{j}\right)$. Let $l(u)$ meet $\partial K$ transversely for the first time $t(u)>\tau$ at $x_{1}(u)=(u, t(u)) \in \partial K_{i_{j}}$. Suitably modifying $V_{j}$ and $\varphi_{j}$ in the interior of $K_{i_{j}}$, we introduce two distributions $\tilde{V}_{j}$ and $\phi_{j}=\tilde{\varphi}_{j}(t, x) \delta\left(t-x_{n}\right)$ so that

$$
\left\{\begin{array}{l}
\square\left(\tilde{V}_{j}+\phi_{j}\right) \in C^{\infty} \quad \text { in } \mathbf{R} \times \Omega, \\
\left.\left(\tilde{V}_{j}+\phi_{j}-v_{j}\right)\right|_{t=\tau}=\left.\frac{\partial}{\partial t}\left(\tilde{V}_{j}+\phi_{j}-v_{j}\right)\right|_{t=\tau}=0 .
\end{array}\right.
$$

We take $\tilde{\varphi}_{j}(t, x) \in C^{\infty}\left(\mathbf{R}^{n+1}\right)$ such that

$$
\tilde{\varphi}_{j}(t, x)= \begin{cases}\varphi_{j}\left(x^{\prime}\right) & \text { for } t \leq t\left(x^{\prime}\right), x_{n} \leq t\left(x^{\prime}\right), x^{\prime} \in \mathscr{O}\left(u_{j}\right), \\ 0 & \text { for } x^{\prime} \notin \mathscr{O}\left(u_{j}\right) \text { or } x_{n}>t\left(x^{\prime}\right)+\varepsilon,\end{cases}
$$

where $\varepsilon>0$ is chosen sufficiently small. Similarly extending $V_{j}$, we arrange (5.5)

$$
\begin{aligned}
& \operatorname{WF}\left(\left.\tilde{V}_{j}\right|_{\mathbf{R} \times \partial K}\right) \cup \mathbf{W F}\left(\left.\frac{\partial \tilde{V}_{j}}{\partial \nu}\right|_{\mathbf{R} \times \partial K}\right) \\
& \subset\left\{\left(t(u), x_{1}(u), \sigma,-\left.\sigma \omega\right|_{T_{x_{1}(u)}(\partial K)}\right) \in T^{*}\left(\mathbf{R} \times \partial K_{i_{j}}^{*}\right) \backslash 0: u \in \mathscr{O}\left(u_{j}\right) \backslash \tilde{\mathscr{O}}\left(u_{j}\right)\right\} .
\end{aligned}
$$


Introduce $W_{j}$ and $w_{j}$ as solutions of the problems:

$$
\begin{gathered}
\left\{\begin{array}{l}
\square W_{j}=0 \text { in } \mathbf{R} \times \Omega, \\
W_{j}+\tilde{V}_{j}=0 \text { on } \mathbf{R} \times \partial K, \\
\left.W_{j}\right|_{t<-\rho_{0}}=0, \quad j=1, \ldots, N,
\end{array}\right. \\
\left\{\begin{array}{l}
\square w_{j}=0 \text { in } \mathbf{R} \times \Omega, \\
w_{j}+\phi_{j}=0 \text { on } \mathbf{R} \times \partial K, \\
\left.w_{j}\right|_{t<-\rho_{0}}=0, \quad j=1, \ldots, N,
\end{array}\right. \\
\left\{\begin{array}{l}
\square w_{j}=0 \text { in } \mathbf{R} \times \Omega, \quad w_{j}=0 \text { on } \mathbf{R} \times \partial K, \\
\left.w_{j}\right|_{t=\tau}=\varphi_{j}\left(x^{\prime}\right) \delta\left(t-x_{n}\right),\left.\quad \frac{\partial w_{j}}{\partial t}\right|_{t=\tau}=\varphi_{j}\left(x^{\prime}\right) \delta^{\prime}\left(\tau-x_{n}\right),
\end{array}\right.
\end{gathered}
$$

for $j=N+1, \ldots, M$.

After this preparation set

$$
\tilde{w}=\sum_{j=1}^{N}\left(w_{j}+\phi_{j}+W_{j}+\tilde{V}_{j}\right)+\sum_{j=N+1}^{M} w_{j}+\sum_{j>M} v_{j}
$$

A simple argument yields $w-\tilde{w} \in C^{\infty}(\mathbf{R} \times \bar{\Omega})$. The singularities of $W_{j}$ are related to the (generalized) rays $\gamma(u)$, with $u$ belonging to $\mathscr{O}\left(u_{j}\right) \backslash \tilde{\mathscr{O}}\left(u_{j}\right)$, which have no segments with direction $\theta$. Consequently,

$$
\begin{array}{r}
\left(\bigcup_{j=1}^{N} \mathrm{WF}\left(\left.\frac{\partial\left(W_{j}+\tilde{V}_{j}\right)}{\partial \nu}\right|_{\mathbf{R} \times \partial K}\right) \cup \bigcup_{j=N+1}^{M} \mathrm{WF}\left(\left.\frac{\partial w_{j}}{\partial \nu}\right|_{\mathbf{R} \times \partial K}\right)\right) \\
\cap\left\{\left(t, x, 1,-\left.\theta\right|_{T_{x}(\partial K)}\right): x \in \partial K,|t| \leq T_{1}\right\}=\varnothing .
\end{array}
$$

Replacing $w$ in (5.1) by $\widetilde{w}$ and using (5.6), we are going to study the asymptotics of the integrals

$$
I_{k, j}(\lambda)=\int_{\mathbf{R}} \int_{\partial K} e^{i \lambda(t-\langle x, \theta\rangle)} \rho_{\varepsilon}^{(k)}\left(\langle x, \theta\rangle-t+T_{0}\right) \frac{\partial}{\partial \nu}\left(w_{j}+\phi_{j}\right) d t d S_{x},
$$

$j=1, \ldots, N$. We have

$$
\frac{\partial \phi_{j}}{\partial \nu}=\frac{\partial \tilde{\varphi}_{j}}{\partial \nu} \delta\left(t-x_{n}\right)-\langle\nu, \omega\rangle \tilde{\varphi}_{j} \delta^{\prime}\left(t-x_{n}\right) \text { on } \mathbf{R} \times \partial K
$$

If $z_{j}$ is a stationary point of $\left.\langle x, \theta-\omega\rangle\right|_{\partial K_{i_{j}}}$ lying on $\partial K_{i_{j}}^{+}$, then $\nu\left(z_{j}\right)=$ $(\theta-\omega) /\|\theta-\omega\|$. Moreover, $\partial \tilde{\varphi}_{j} / \partial \nu$ and $\partial \tilde{\varphi}_{j} / \partial t$ vanish on some neighborhood of $z_{j}$ in $\partial K$. Therefore a stationary phase argument implies

$$
I_{k, j}(\lambda)=\int_{\mathbf{R}} \int_{\partial K} e^{i \lambda(t-\langle x, \theta\rangle)} \rho_{\varepsilon}^{(k)}\left(\langle x, \theta\rangle-t+T_{0}\right) \mathscr{B} w_{j} d t d S
$$


$\bmod \mathscr{O}\left(|\lambda|^{-m}\right)$ for every $m$ with $\mathscr{B} w_{j}=\left.(\partial / \partial \nu-\langle\nu, \theta\rangle \partial / \partial t) w_{j}\right|_{\mathbf{R} \times \partial K}$. Here we have used the equality

$$
\begin{aligned}
\int_{\partial K} e^{i \lambda\langle x, \omega-\theta\rangle}\langle\nu, \theta & +\omega\rangle \rho_{\varepsilon}^{(k)}\left(\langle x, \theta-\omega\rangle+T_{0}\right) d S_{x} \\
& =\int_{K} \frac{\partial}{\partial(\theta+\omega)}\left[e^{i \lambda\langle x, \omega-\theta\rangle} \rho_{\varepsilon}^{(k)}\left(\langle x, \theta-\omega\rangle+T_{0}\right)\right] d x=0 .
\end{aligned}
$$

Fix $M_{0}+1 \leq j \leq N$. The wave front $\operatorname{WF}\left(\mathscr{B} w_{j}\right)$ is related to the rays $\gamma(u)$, $u \in \mathscr{O}\left(u_{j}\right)$. As we mentioned above, the first $r_{0}$ reflection points of these rays are proper ones. Then by the construction in [16], we take $w_{j}$ in the form

$$
w_{j}=\sum_{p=1}^{r_{0}-1} w_{p, j}+w_{j}^{\prime} .
$$

By using (5.3), the singularities of $\mathscr{B} w_{p, j}$ are related to the finite segments $\left[x_{p}(u), x_{p+1}(u)\right]$ meeting $\partial K$ transversally. Extending $w_{p, j}$ in the interior of $\partial K$ and repeating the argument in [16] with the outgoing Green function, we conclude that $w_{p, j}$ does not contribute to the asymptotic of $I_{k, j}(\lambda)$. On the other hand, the singularities of $\mathscr{B} w_{j}^{\prime}$ are connected with the segments issued from $x_{p}(u), p=r_{0}, \ldots, q$ which have no directions $\theta$. Consequently, $I_{k, j}(\lambda)=\mathscr{O}\left(|\lambda|^{-m}\right), \forall m$, for $M_{0}+1 \leq j \leq N$.

To study $I_{k, j}(\lambda), j=1, \ldots, M_{0}$, notice that $\mathrm{WF}\left(\mathscr{B} w_{j}\right)$ is related to ordinary reflecting rays issued from $\mathrm{WF}\left(\left.\phi_{j}\right|_{\mathbf{R} \times \partial K_{i_{j}}^{+}}\right)$. Thus we are in a position to apply the results of Petkov [16] for $I_{k, j}(\lambda)$. The arguments in [16] with trivial modifications work for dimensions $n \geq 3, n$ odd. In particular, instead of Lemmas 1 and 2 on p. 321 of [16] we obtain directly the following.

Lemma 5.1. Let $G, P$, and $Q$ be $(n-1) \times(n-1)$ matrices. Then

$$
\operatorname{det}\left(\begin{array}{ccc}
-G & 0 & -I \\
0 & P & I \\
-I & I & Q
\end{array}\right)=\operatorname{det}(G Q P+P-G) .
$$

Proof. First assume $G$ invertible. Then

$$
\begin{array}{r}
\operatorname{det}\left(\begin{array}{ccc}
-G & 0 & -I \\
0 & P & I \\
-I & I & Q
\end{array}\right) \cdot \operatorname{det} G^{-1}=\operatorname{det}\left(\begin{array}{ccc}
-I & 0 & -I \\
0 & P & I \\
-G^{-1} & I & Q
\end{array}\right) \\
=\operatorname{det}\left(\begin{array}{cc}
-I & P \\
-G^{-1}-Q & I
\end{array}\right)=\operatorname{det}\left(G^{-1} P+Q P-I\right),
\end{array}
$$

which implies (5.6). In the case $\operatorname{det} G=0$, we choose $\varepsilon_{0}>0$ so that $G_{\varepsilon}=G+\varepsilon I$ is invertible for $0<\varepsilon<\varepsilon_{0}$. Applying (5.6) for $G_{\varepsilon}$ and letting $\varepsilon \rightarrow 0$, we complete the proof.

As we proved in $\S 3$, the map $d J_{\gamma}$ (see $\S 1$ ) is invertible at the point $A_{\gamma}$, where $\gamma$ hits $Z_{\omega}$. Moreover, the sojourn times of the different $(\omega, \theta)$-rays are 
different for $\theta \in \mathscr{R}(\omega)$. Therefore, from the asymptotic of $I_{k, j}(\lambda)$, given in [16], we get

$$
\text { singsupp } s(t, \theta, \omega) \cap\{|t| \leq T\}=\left\{-T_{\gamma}: \gamma \in \mathscr{L}_{\omega, \theta},\left|T_{\gamma}\right| \leq T\right\} .
$$

Since $T$ is arbitrary, we obtain (1.4). The form of the leading singularity at $-T_{\gamma}$ follows from [16]. This completes the proof of Theorem 1.1.

\section{EXISTENCE OF $(\omega, \theta)$-RAYS AND ASYMPTOTICS OF $T_{m}^{1 l}$}

Throughout this section we assume that $K=\bigcup_{i=1}^{s} K_{i}$ satisfies condition (H) introduced in $\S 1$. Let $\mathscr{R}(\omega)=\mathscr{R}_{1}(\omega) \cap \mathscr{R}_{2}(\omega)$, where $\mathscr{R}_{1}(\omega)$ and $\mathscr{R}_{2}(\omega)$ are the residual subsets of $S^{n-1}$ defined in $\S 4$.

Definition 6.1. Let $\alpha=\left(i_{1}, \ldots, i_{k}\right)$ be a configuration. We say that a pair $(\omega, \theta)$ of unit vectors satisfies the conditions of visibility with respect to $\alpha$ if $\theta \in \mathscr{R}(\omega)$ and the following conditions hold:

(a) for every $x \in \partial K_{i_{1}}$ the ray starting at $x$ with direction $-\omega$ (resp. $\omega$ ) has no common point with $K \backslash K_{i_{1}}$ (resp. $K_{i_{2}}$ );

(b) for every $x \in \partial K_{i_{k}}$ the ray starting at $x$ with direction $\theta$ (resp. $-\theta$ ) has no common point with $K \backslash K_{i_{k}}$ (resp. $\left.K_{i_{k-1}}\right)$.

Lemma 6.2. For every configuration $\alpha=\left(i_{1}, \ldots, i_{k}\right)$ there exist $\omega, \theta \in S^{n-1}$ such that $(\omega, \theta)$ satisfies the conditions of visibility with respect to $\alpha$.

Proof. Take a hyperplane $A$ which separates $K_{i_{1}}$ and $K_{i_{2}}$ and such that $A$ is tangent to $K_{i_{1}}$ at some point $x$, while $A$ is tangent to $K_{i_{2}}$ at some point $y$. Set $\omega^{\prime}=(y-x) /\|y-x\|$ and denote by $Z$ an arbitrary hyperplane orthogonal to $\omega^{\prime}$ such that the open half-space, determined by $Z$ and having $\omega^{\prime}$ as an inward normal, contains $K$. Consider the convex cone $C=\left\{y+t(u-y): u \in K_{i_{1}}\right.$, $t \geq 0\}$. It is easy to see that the orthogonal projection of $K_{i_{1}}$ on $Z$ is contained in $C$. Therefore, condition (H) implies

$$
\left\{u-t \omega^{\prime}: t \geq 0\right\} \cap\left(K \backslash K_{i_{1}}\right)=\varnothing, \quad u \in K_{i_{1}} .
$$

Indeed, suppose there are $u \in K_{i_{1}}, t>0$, and $j \neq i_{1}$ so that $v=u-t \omega^{\prime} \in K_{j}$. Let $u^{\prime}$ be the orthogonal projection of $u$ (and therefore of $v$ ) on $Z$. Since $u, u^{\prime} \in C$, we have $v \in C$. On the other hand, the definition of $C$ shows that the segment $[y, v]$ contains a point of $K_{i_{1}}$ which is a contradiction with condition (H). Hence (6.1) holds and the compactness of $K \backslash K_{i_{1}}$ implies the existence of a number $\varepsilon>0$ so that

$$
\{u-t \omega: t \geq 0\} \cap\left(K \backslash K_{i_{1}}\right)=\varnothing, \quad u \in K_{i_{1}},
$$

whenever $\left\|\omega-\omega^{\prime}\right\|<\varepsilon$. Choose $\omega$ with $\left\|\omega-\omega^{\prime}\right\|<\varepsilon$ and $\langle-\omega, \nu(x)\rangle>0$. Then it is clear that condition (a) of Definition 6.1 holds. 
Since $\mathscr{R}(\omega)$ is dense in $S^{n-1}$, in a similar way we can find $\theta \in \mathscr{R}(\omega)$ satisfying (b). This completes the proof of the lemma.

Fix a configuration $\alpha=\left(i_{1}, \ldots, i_{k}\right)$ with $i_{1} \neq i_{k}$ and an integer $l=$ $1, \ldots, k$. For $q=0,1, \ldots$ set

$$
\alpha_{q, 1}=(\underbrace{i_{1}, \ldots, i_{k} ; \cdots ; i_{1}, \ldots, i_{k}}_{q} ; i_{1}, \ldots, i_{l}) .
$$

Proposition 6.3. Let $(\omega, \theta)$ satisfy the conditions of visibility with respect to $\alpha_{1, l}$. Then for every integer $q \geq 0$ there exists an $(\omega, \theta)$-ray of type $\alpha_{q, 1}$.

Proof. Fix $q \geq 0$ and two hyperplanes $Z_{\omega}, Z_{\theta}$ as in the text preceding Theorem 4.2. Set $m=q k+l, D=Z_{\omega} \times \partial K_{i_{1}} \times \cdots \times \partial K_{i_{m}} \times Z_{\theta}$ and define $F: D \rightarrow \mathbf{R}$ by

$$
F(\xi)=\left\|z_{1}-x_{1}\right\|+\sum_{j=1}^{m-1}\left\|x_{j}-x_{j+1}\right\|+\left\|x_{m}-z_{2}\right\|,
$$

where $\xi=\left(z_{1} ; x_{1}, \ldots, x_{m} ; z_{2}\right) \in D$. Since $F$ is continuous, it is clear that there exists $\bar{\xi}=\left(\bar{z}_{1} ; \bar{x}_{1}, \ldots, \bar{x}_{m} ; \bar{z}_{2}\right) \in D$ with $F(\bar{\xi})=\min F$. Moreover, $\bar{z}_{1}=\pi_{\omega}\left(\bar{x}_{1}\right)$ and $\bar{z}_{2}=\pi_{\theta}\left(\bar{x}_{m}\right)$. Then from condition (a) we deduce that the segment $\left[\bar{z}_{1}, \bar{x}_{2}\right]$ has no common point with $K_{i_{1}}$. For $c>0$ consider the rotative ellipsoid

$$
E_{c}=\left\{x \in \mathbf{R}^{n}:\left\|\bar{z}_{1}-x\right\|+\left\|x-x_{2}\right\| \leq c\right\} .
$$

Take the minimal $c>0$ with $E_{c} \cap K_{i_{1}} \neq \varnothing$. Therefore, $E_{c}$ will be tangent to $\partial K_{i_{1}}$ at some $\bar{x}_{1}^{\prime}$. In view of $F(\bar{\xi})=\min F(\xi)$ we get $\bar{x}_{1}=\bar{x}_{1}^{\prime}$. Thus the segments $\left[\bar{z}, \bar{x}_{1}\right]$ and $\left[\bar{x}_{1}, \bar{x}_{2}\right]$ satisfy the law of reflection at $x$.

Repeating this argument and exploiting conditions (H) and (b), we deduce that $\bar{x}_{1}, \ldots, \bar{x}_{m}$ are the successive reflection points of an $(\omega, \theta)$-ray of type $\alpha_{q, l}$. This proves the proposition.

Remark. In [23] we announced the existence of $(\omega, \theta)$-rays under weaker conditions than those given above. However, there are counterexamples showing that this statement in [23] is not true.

Up to the end of this section we will assume that $\alpha$ and $l$ are fixed and $(\omega, \theta)$ are fixed too so that the conditions of visibility with respect to $\alpha_{1, l}$ are satisfied. This assumption combined with $(\mathbf{H})$ implies the existence of a constant $\kappa_{1}=\kappa_{1}(\alpha, \omega)>0$ such that if $\gamma(z)$ is a ray issued from $z \in Z_{\omega}$ with direction $\omega$ and having successive reflection points $x_{1} \in \partial K_{i_{1}}, x_{2} \in \partial K_{i_{2}}$, $x_{3}, \ldots, x_{p}$, then

$$
\cos \varphi_{j} \geq \kappa_{1}, \quad j=1, \ldots, p-1,
$$

where $\varphi_{j}$ is the angle between $\nu\left(x_{j}\right)$ and $x_{j} \overrightarrow{x_{j+1}} \quad(1 \leq j \leq p-1)$.

Let $V_{0} \subset Z_{\omega}$ be the set of points such that every ray $\gamma(z)$ issued from $z \in V_{0}$ with direction $\omega$ has successive reflection points

$$
u_{q k+j}^{(z)} \in \partial K_{i_{j}}, \quad 0 \leq q \leq m, 1 \leq j \leq k(1 \leq j \leq l \text { for } q=m) .
$$


Of course $V_{0}$ depends on $m, l$ but we will not mention this explicitly. Let $\varphi(x)$ be a real smooth function defined in $\mathscr{U}$ so that $|\nabla \varphi|=1$. We say that $\varphi$ satisfies condition (P) in $\mathscr{U}$ if the principal curvatures of $\mathscr{L}_{\varphi}(x)=\{y \in \mathscr{U}$ : $\varphi(y)=\varphi(x)\}$ with respect to $-\nabla \varphi$ are nonnegative for all $x \in \mathscr{U}$.

Choose $\varphi_{0}(x)=\langle x, \omega\rangle$ and $\mathscr{U}_{0}=\left\{y+\tau \omega, y \in V_{0}: \tau \geq 0\right\}$. Obviously, $\varphi_{0}$ satisfies $(\mathrm{P})$ in $\mathscr{U}_{0}$. Let $\varphi_{1}(x)$ be a function so that $\left|\nabla \varphi_{1}\right|=1$ and

$$
\varphi_{1}(x)=\varphi_{0}(x), \quad \frac{\partial \varphi_{1}}{\partial \nu}=-\frac{\partial \varphi_{0}}{\partial \nu} \quad \text { on } V_{1}=\mathscr{U}_{0} \cap \partial K_{i_{1}}^{+}
$$

We extend $\varphi_{1}$ on $\mathscr{U}_{1}=\bigcup_{y \in V_{1}}\left\{y+\tau \nabla \varphi_{1}(y): \tau \geq 0\right\}$ so that $\varphi_{1}$ satisfies (P) in $\mathscr{U}_{1}$ (see [9]). Following this procedure we define successive functions $\varphi_{0}, \varphi_{1}, \varphi_{2}, \ldots, \varphi_{q k+j}$ satisfying $(\mathrm{P})$ respectively in $\mathscr{U}_{0}, \mathscr{U}_{1}, \mathscr{U}_{2}, \ldots, \mathscr{U}_{q k+j}$, where

$$
\mathscr{U}_{q k+j}=\bigcup_{y \in V_{q k+j}}\left\{y+\tau \nabla \varphi_{q k+j}(y): \tau \geq 0\right\}, \quad V_{q k+j} \subset \partial K_{i_{j}} .
$$

Let $\gamma_{1}$ and $\gamma_{2}$ be two ordinary reflecting rays issued respectively from $y_{0}, z_{0}$ $\in V_{0}$ with direction $\omega$ and having successive reflection points

$$
y_{q k+j}, \quad z_{q k+j} \in \partial K_{i_{j}}, \quad 0 \leq q \leq m, 1 \leq j \leq k(1 \leq j \leq l \text { for } q=m) .
$$

The properties of $\varphi_{0}, \ldots, \varphi_{q k+j}$ combined with the proof of Proposition 3.8 in [9] yield

Lemma 6.4. There exist constants $C>0,0<\delta<1$, independent of $\gamma_{1}, \gamma_{2}, l$ so that

$$
\left\|y_{i}-z_{i}\right\| \leq C \delta^{m k+l-i}, \quad i=0, \ldots, m k+l .
$$

Constructing rays with $m$ reflections, letting $m \rightarrow \infty$, and exploiting (6.2), it is easy to find a unique ray $\gamma^{\infty}(\omega)$ starting at $x_{0}^{\infty} \in Z_{\omega}$ with direction $\omega$ and having infinite number reflection points $x_{q k+j}^{\infty} \in \partial K_{i_{j}}, q \geq 0,1 \leq j \leq k$. The properties of the dispersive billiards [24, 25] (see also Lemma 5.4 in [9]) yield

$$
\left\|x_{q k+j}^{\infty}-\tilde{x}_{j}\right\| \leq C_{1} \delta^{q k+j}, \quad q \geq 0,1 \leq j \leq k,
$$

with $C_{1}$ independent of $q$ and $j$.

Set $d_{j}=\sum_{p=1}^{j}\left\|\tilde{x}_{p+1}-\tilde{x}_{p}\right\|, \quad 1 \leq j \leq k, d_{\alpha}=d_{k}, L_{m}^{\infty}=\left\langle x_{1}^{\infty}, \omega\right\rangle+$ $\sum_{p=1}^{m}\left\|x_{p+1}^{\infty}-x_{p}^{\infty}\right\|$. Applying (6.3) for $q \geq 0, r \geq 0$, we have

$$
\left|\left(L_{(q+r) k+j}^{\infty}-(q+r) d_{\alpha}-d_{j}\right)-\left(L_{q k+j}^{\infty}-q d_{\alpha}-d_{j}\right)\right| \leq 2 C_{1} \sum_{p=1}^{r k+1} \delta^{q k+p+j} \leq C_{2} \delta^{q} .
$$

Fixing $j$ and introducing $L_{\alpha, \omega, j}^{1}=\lim _{q \rightarrow \infty}\left(L_{q k+j}^{\infty}-q d_{\alpha}-d_{j}\right)$, we get

$$
L_{q k+j}^{\infty}=q d_{\alpha}+d_{j}+L_{\alpha, \omega, j}^{1}+O\left(\delta^{q}\right), \quad q \rightarrow \infty .
$$


As above, there exists a unique ray $\gamma^{\infty}(-\theta)$ starting at some $y_{0}^{\infty} \in Z_{\theta}$ with direction $-\theta$ and having an infinite number of reflection points $y_{q k+r}^{\infty} \in \partial K_{j_{r}}$, $q \geq 0,1 \leq r \leq k$, where $\left(j_{1}, \ldots, j_{k}\right)=\left(i_{l}, i_{l-1}, \ldots, i_{1}, i_{k}, i_{k-1}, \ldots, i_{l+1}\right)$. Therefore, we obtain

$$
G_{q k}^{\infty}=-\left\langle y_{1}^{\infty}, \theta\right\rangle+\sum_{p=1}^{m}\left\|y_{p+1}^{\infty}-y_{p}^{\infty}\right\|=q d_{\alpha}+L_{\alpha,-\theta}^{1}+O\left(\delta^{q}\right), \quad q \rightarrow \infty
$$

with some constant $L_{\alpha,-\theta}^{l}$.

Now let $\gamma_{m}^{1 l}$ be an ordinary $(\omega, \theta)$-ray with sojourn time $T_{m}^{1 l}$ and $m=q k+l$ reflection points $x_{p k+j}^{(m)} \in \partial K_{i_{j}}, 0 \leq p \leq q, 1 \leq j \leq k \quad(1 \leq j \leq l$ for $p=q)$. Set

$L_{p}^{(m)}=\left\langle x_{1}^{(m)}, \omega\right\rangle+\sum_{r=1}^{p}\left\|x_{r+1}^{(m)}-x_{r}^{(m)}\right\|, \quad G_{p}^{(m)}=-\left\langle x_{m}^{(m)}, \theta\right\rangle+\sum_{r=p+1}^{m-1}\left\|x_{r+1}^{(m)}-x_{r}^{(m)}\right\|$,

and take $p=[q / 2] k+l-1$. Applying (6.2) twice for the reflection points of $\gamma_{m}^{1 l}, \gamma^{\infty}(\omega)$ as well as for those of $\gamma_{m}^{1 l}, \gamma^{\infty}(-\theta)$, we obtain

$$
\begin{aligned}
\left|L_{p}^{(m)}-L_{p}^{\infty}\right|+\mid G_{p}^{(m)} & -G_{q-[q / 2] k} \mid \\
& \leq 2 \sum_{r=1}^{p+1}\left\|x_{r}^{(m)}-x_{r}^{(m)}\right\|+2 \sum_{q=1}^{m-p}\left\|x_{m-r+1}^{(m)}-y_{r}^{\infty}\right\| \leq C_{3} \delta^{q} .
\end{aligned}
$$

Since $T_{m}^{1 l}=L_{p}^{(m)}+G_{p}^{(m)}$, by (6.4) and (6.5) we get

Theorem 6.5. Assume $(\mathrm{H})$ is fulfilled. Let $\alpha=\left(i_{1}, \ldots, i_{k}\right), i_{1} \neq i_{k}$, be $a$ configuration and let $(\omega, \theta)$ satisfy the conditions of visibility with respect to $\alpha_{1, l}$. Then we have the asymptotic

$$
T_{q k+l}^{1 l}=q d_{\alpha}+L_{\alpha, \omega, \theta}^{1 l}+O\left(\delta^{q}\right), \quad q \rightarrow \infty,
$$

where $L_{\alpha, \omega, \theta}^{1 l}=L_{\alpha, \omega, l-1}^{1}+L_{\alpha,-\theta}^{l}+d_{l-1}, \quad d_{0}=0, L_{\alpha, \omega, 0}^{1}=L_{\alpha, \omega, k}^{1}$.

Corollary 6.6. Let $K=K_{1} \cup K_{2}, d=\operatorname{dist}\left(K_{1}, K_{2}\right)$. Assume $(\omega, \theta)$ satisfies the conditions of visibility with respect to $\alpha=(1,2)$. Let $\gamma_{m}^{i j}$ be the $(\omega, \theta)$ ray with sojourn time $T_{m}^{i j}$ and $m$ reflection points $\left\{x_{p}^{(m)}\right\}$, where $x_{1}^{(m)} \in \partial K_{i}$, $x_{m}^{(m)} \in \partial K_{j}$. Then we have

$$
T_{2 q+i+j-1}^{i j}=2 q d+L_{\omega, \theta}^{i j}+O\left(\delta^{q}\right), \quad q \rightarrow \infty, i, j=1,2 .
$$

Note that a particular case of (6.7) for two disjoint balls has been obtained by Nakamura and Soga [15]. The asymptotic (6.6) is similar to this for the lengths of the periodic reflecting rays in bounded strictly convex domains $\mathscr{O} \subset \mathbf{R}^{2}$ (see $[2,12])$.

\section{ASYMPTOTIC BEHAVIOR OF $\left|c_{m}^{1 !}\right|$}

Throughout this section we assume $(H)$ is fulfilled and we use freely the notation of $\S 6$. Fix $\omega \neq \theta$ and a configuration $\alpha=\left(i_{1}, \ldots, i_{k}\right)$ with $i_{1} \neq i_{k}$. 
Assume $l$ is fixed and $(\omega, \theta)$ satisfies the conditions of visibility with respect to $\alpha_{1, l}$. Then it is easy to find a constant $\kappa=\kappa(K, \omega, \theta)>0$ such that if $\gamma$ is an $(\omega, \theta)$-ray with successive reflection points $x_{p k+j} \in K_{i_{j}}, 1 \leq p \leq q$, $1 \leq j \leq k$, then

$$
\cos \varphi_{j} \geq \kappa, \quad j=1, \ldots, q k+l,
$$

$\varphi_{j}$ being the angle between $\nu\left(x_{j}\right)$ and $x_{j} \vec{x}_{j+1}$ (resp. $\theta$ for $j=q k+l$ ). Set

$$
d=\operatorname{diam} K, \quad d^{\prime}=\min _{i \neq j} \operatorname{dist}\left(K_{i}, K_{j}\right), \quad d_{0}=1 / d^{\prime} .
$$

Since $K_{i}$ are strictly convex, there exist constants $\mu_{2}>\mu_{1}>0$ so that

$$
\mu_{1}\langle v, v\rangle \leq\left\langle G_{x} v, v\right\rangle \leq \mu_{2}\langle v, v\rangle, \quad v \in T_{x} \partial K,
$$

where $G_{x}$ is the differential of the Gauss map of $\partial K$ at $x$.

Let $x \in \partial K_{i}, y \in \partial K_{j}, i \neq j$. Suppose the segment $s_{x, y}=[x, y] \subset \Omega$ is transverse to both $\partial K_{i}$ and $\partial K_{j}$. Let $\bar{x}$ be a point lying on the line joining $x$ and $y$ such that $\|x-\bar{x}\|=1$. Let $\sqcap$ be the hyperplane orthogonal to $s_{x, y}$ and passing through $\bar{x}$. Seting $e=(y-x) /\|y-x\|$, denote by $\pi$ the projection $\sqcap \rightarrow T_{x} \partial K$ along the direction $-e$. Introduce the symmetric operator $\widetilde{\psi}: \sqcap \rightarrow \Pi$ by

$$
\langle\tilde{\psi} u, u\rangle=2\langle e, \nu(x)\rangle\left\langle G_{x} \pi u, \pi u\right\rangle, \quad u \in \Pi \text {. }
$$

We will say that $\tilde{\psi}$ is related to $s_{x, y}$. It follows easily that

$$
\operatorname{spec} \tilde{\psi} \subset\left[2 \mu_{1}\langle\nu(x), e\rangle, 2 \mu_{2}\langle\nu(x), e\rangle^{-1}\right]
$$

Now we will prove a technical lemma which will be applied several times in this section. We need to introduce some assumptions and notation. Let $\beta=\left(\overline{i_{1}}, \ldots, \overline{i_{p}}\right)$ be a configuration and let $x_{j}, x_{j}^{\prime} \in \partial K_{\overline{i_{j}}}, j=1, \ldots, p$, be points so that

$$
\left\|x_{j}-x_{j}^{\prime}\right\|<D a^{j}, \quad j=1, \ldots, p,
$$

with some constants $D>0, a>0$. Assume $\left\langle e_{j}, \nu\left(x_{j}\right)\right\rangle \geq \kappa,\left\langle e_{j}^{\prime}, \nu\left(x_{j}^{\prime}\right)\right\rangle \geq \kappa$, where $e_{j}=\left(x_{j+1}-x_{j}\right) /\left\|x_{j+1}-x_{j}\right\|, e_{j}^{\prime}=\left(x_{j+1}^{\prime}-x_{j}^{\prime}\right) /\left\|x_{j+1}^{\prime}-x_{j}^{\prime}\right\|, j=1, \ldots, p$. According to Lemma $\mathbf{A}$ in the Appendix, for every $j=1, \ldots, p$ there exists an isometry $A_{j}: \mathbf{R}^{n} \rightarrow \mathbf{R}^{n}$ such that $A_{j}\left(\Pi_{j}^{\prime}\right)=\Pi_{j}$ and

$$
\left\|A_{j}-I\right\|<C_{1} D(1+a) a^{j}, \quad\left\|\tilde{\psi}_{j}-A_{j} \tilde{\psi}_{j}^{\prime} A_{j}^{-1}\right\|<C_{1} D(1+a) a^{j}
$$

Here $\tilde{\psi}_{j}: \Pi_{j} \rightarrow \Pi_{j}$ and $\tilde{\psi}_{j}^{\prime}: \Pi_{j}^{\prime} \rightarrow \Pi_{j}^{\prime}$ are the operators related to the segments $s_{j}=\left[x_{j}, x_{j+1}\right]$ and $s_{j}^{\prime}=\left[x_{j}^{\prime}, x_{j+1}^{\prime}\right]$. Let $M_{1}: \Pi_{1} \rightarrow \Pi_{1}$ and $M_{1}^{\prime}: \Pi_{1}^{\prime} \rightarrow \Pi_{1}^{\prime}$ be arbitrary symmetric positive definite linear operators. Set

$$
M_{i}=\sigma_{i} M_{i-1}\left(I+\lambda_{i} M_{i-1}\right)^{-1} \sigma_{i}+\tilde{\psi}_{i}, \quad i=2, \ldots, p,
$$


where $\lambda_{i}=\left\|x_{i-1}-x_{i}\right\|$ and $\sigma_{i}$ is the symmetry with respect to $\Pi_{i}$. Let $M_{i}^{\prime}$ be the map defined by (7.6) replacing $\tilde{\psi}_{i}, \sigma_{i}, \lambda_{i}, x_{j}$ by $\tilde{\psi}_{i}^{\prime}, \sigma_{i}^{\prime}, \lambda_{i}^{\prime}, x_{i}^{\prime}$, respectively. Finally, set

$$
b=\left(1+2 \mu_{1} \kappa d^{\prime}\right)^{-1}, \quad a_{1}= \begin{cases}a & \text { for } a \geq 1, \\ \max (a, b) & \text { for } a<1 .\end{cases}
$$

Lemma 7.1. Under the assumptions and notation above there exist constants $E>0, E^{\prime}>0$, depending only on $\kappa, K$, and a such that

$$
\begin{gathered}
\left\|M_{j}-A_{j} M_{j}^{\prime} A_{j}^{-1}\right\|<D E a_{1}^{j}+b^{2(j-v)}\left\|M_{v}-A_{v} M_{v}^{\prime} A_{v}^{-1}\right\| \\
\left|\ln \operatorname{det}\left(I+\lambda_{j+1} M_{j}\right)\left(I+\lambda_{j+1}^{\prime} M_{j}^{\prime}\right)^{-1}\right| \\
<D E^{\prime} a_{1}^{j}+(n-1) d b^{2(j-v)+1}\left\|M_{v}-A_{v} M_{v}^{\prime} A_{v}^{-1}\right\|
\end{gathered}
$$

for $1 \leq v \leq j \leq p$.

Proof. First notice that $\left|\lambda_{i}-\lambda_{i}^{\prime}\right|<D(1+a) a^{i}$. Moreover, $\min \operatorname{spec} M_{i-1} \geq$ min spec $\widetilde{\psi}_{i-1}(i \geq 2)$ implies (see subsection 2.1 )

$$
\left\|\left(I+\lambda_{i} M_{i-1}\right)^{-1}\right\| \leq b, \quad\left\|M_{i-1}\left(I+\lambda_{i} M_{i-1}\right)^{-1}\right\| \leq 1 / \lambda_{i} \leq d_{0} .
$$

Introduce $L_{i}=A_{i} M_{i}^{\prime} A_{i}^{-1}: \Pi_{i} \rightarrow \Pi_{i}$. Then $\sigma_{i}^{\prime}=A_{i}^{-1} \sigma_{i} A_{i}$ and we obtain

$$
L_{i}=\sigma_{i} B_{i} L_{i-1}\left(I+\lambda_{i}^{\prime} L_{i-1}\right)^{-1} B_{i}^{-1} \sigma_{i}+A_{i} \widetilde{\psi}_{i}^{\prime} A_{i}^{-1},
$$

where $B_{i}=A_{i} A_{i-1}^{-1}$. Combining (7.5) with the inequality

$$
\left\|X-B_{i} Y B_{i}^{-1}\right\| \leq 2\|X\| \cdot\left\|I-B_{i}\right\|+\|X-Y\|
$$

we deduce

$$
\begin{aligned}
\left\|M_{i}-L_{i}\right\| \leq & \left\|M_{i-1}\left(I+\lambda_{i} M_{i-1}\right)^{-1}-B_{i} L_{i-1}\left(I+\lambda_{i}^{\prime} L_{i-1}\right)^{-1} B_{i}^{-1}\right\| \\
& +\left\|\widetilde{\psi}_{i}-A_{i} \widetilde{\psi}_{i}^{\prime} A_{i}^{-1}\right\|<C_{1} D(1+a) a^{i}+2 C_{1} d_{0} D(1+a)^{2} a^{i-1} \\
& +\left\|M_{i-1}\left(I+\lambda_{i} M_{i-1}\right)^{-1}-L_{i-1}\left(I+\lambda_{i}^{\prime} L_{i-1}\right)^{-1}\right\| .
\end{aligned}
$$

On the other hand, using subsection 2.4 , we get

$$
\begin{aligned}
& \left\|M_{i-1}\left(I+\lambda_{i} M_{i-1}\right)^{-1}-L_{i-1}\left(I+\lambda_{i}^{\prime} L_{i-1}\right)^{-1}\right\| \\
& \quad \leq\left|\lambda_{i}-\lambda_{i}^{\prime}\right| \cdot\left\|\left(I+\lambda_{i} M_{i-1}\right)^{-1} M_{i-1}\right\| \cdot\left\|L_{i-1}\left(I+\lambda_{i}^{\prime} L_{i-1}\right)^{-1}\right\| \\
& \quad+b^{2}\left\|M_{i-1}-L_{i-1}\right\| \\
& \quad<D d_{0}^{2}(1+a) a^{i}+b^{2}\left\|M_{i-1}-L_{i-1}\right\| .
\end{aligned}
$$

Therefore,

$$
\left\|M_{i}-L_{i}\right\|<D E^{\prime \prime} a^{i}+b^{2}\left\|M_{i-1}-L_{i-1}\right\|, \quad i=2, \ldots, p,
$$

with $E^{\prime \prime}=(1+a)\left(C_{1}+2 d_{0}(1+a) a^{-1} C_{1}+d_{0}^{2}\right)>0$. 
By induction on $j$ we get

$$
\left\|M_{j}-L_{j}\right\|<D E^{\prime \prime} \sum_{r=0}^{j-v-1} a^{j-r} b^{2 r}+b^{2(j-v)}\left\|M_{v}-L_{v}\right\|
$$

for $1 \leq v \leq j \leq p$.

Case 1. $a \geq 1$. Then $a>b^{2}$ and (7.10) yields

$$
\begin{aligned}
\left\|M_{j}-L_{j}\right\| & <D E^{\prime \prime} a^{j} \sum_{r=0}^{j-v-1}\left(b^{2} / a\right)^{r}+b^{2(j-v)}\left\|M_{v}-L_{v}\right\| \\
& <D E^{\prime \prime} a^{j}\left(1-b^{2} / a\right)^{-1}+b^{2(j-v)}\left\|M_{v}-L_{v}\right\| .
\end{aligned}
$$

In this case we set $E=E^{\prime \prime} a\left(a-b^{2}\right)^{-1}$.

Case 2. $a<1$. Taking $a_{1}=\max (a, b)$, by (7.10) we have

$$
\begin{aligned}
\left\|M_{j}-L_{j}\right\| & <D E^{\prime \prime} \sum_{r=0}^{j-v-1} a_{1}^{j+r}+b^{2(j-v)}\left\|M_{v}-L_{v}\right\| \\
& <D E^{\prime \prime}\left(1-a_{1}\right)^{-1} a_{1}^{j}+b^{2(j-v)}\left\|M_{v}-L_{v}\right\| .
\end{aligned}
$$

Then we set $E=E^{\prime \prime}\left(1-a_{1}\right)^{-1}$.

Consequently, we have proved (7.8).

To verify (7.9), we use the estimate

$$
\begin{aligned}
\operatorname{det}\left[\left(I+\lambda_{i+1} M_{i}\right)\left(I+\lambda_{i+1}^{\prime} M_{i}^{\prime}\right)^{-1}\right] \leq\left(1+\left\|I-\left(I+\lambda_{i+1} M_{i}\right)\left(I+\lambda_{i+1}^{\prime} L_{i}^{\prime}\right)^{-1}\right\|\right)^{n-1} \\
\quad=\left(1+\left\|\left(\lambda_{i+1}^{\prime} L_{i}-\lambda_{i+1} M_{i}\right)\left(I+\lambda_{i+1}^{\prime} L_{i}\right)^{-1}\right\|\right)^{n-1} \\
\quad \leq\left(1+\frac{\left|\lambda_{i+1}^{\prime}-\lambda_{i+1}\right|}{\lambda_{i+1}^{\prime}}+b \lambda_{i+1}\left\|M_{i}-L_{i}\right\|\right)^{n-1} \\
\quad<\left(1+D(1+a) d_{0} a^{i+1}+b d\left\|M_{i}-L_{i}\right\|\right)^{n-1} .
\end{aligned}
$$

Similar equality holds for $\operatorname{det}\left[\left(I+\lambda_{i+1} M_{i}\right)^{-1}\left(I+\lambda_{i+1}^{\prime} M_{i}^{\prime}\right)\right]$ and we obtain

$$
\begin{aligned}
& \left|\ln \operatorname{det}\left(I+\lambda_{i+1} M_{i}\right)-\ln \operatorname{det}\left(I+\lambda_{i+1}^{\prime} M_{i}^{\prime}\right)\right| \\
& \quad<(n-1) \ln \left(1+D d_{0}(1+a) a^{i+1}+b d\left\|M_{i}-L_{i}\right\|\right) \\
& \quad<(n-1)\left(D d_{0}+(1+a) a^{i+1}+b d\left\|M_{i}-L_{i}\right\|\right) .
\end{aligned}
$$

Applying (7.8) for $j=i$, we arrange (7.9) with

$$
E^{\prime}=(n-1) \cdot\left(\left(a_{1}^{2}+a_{1}\right) d_{0}+b d E\right) \text {. }
$$

This completes the proof of Lemma 7.2.

Let $\tilde{x}_{1}, \ldots, \tilde{x}_{k}$ be the reflection points of the periodic ray $\gamma_{\alpha}$ related to $\alpha$, where $\tilde{x}_{j} \in \partial K_{i j}$ for $j=1, \ldots, k$. For convenience we set $\tilde{x}_{m}=\tilde{x}_{j}$ for $m=p k+j, 1 \leq j \leq k$. Let $\tilde{\tilde{\psi}}_{j}: \tilde{\Pi}_{j} \rightarrow \tilde{\Pi}_{j}$ be the operator related to 
$\left[\tilde{x}_{j}, \tilde{x}_{j+1}\right]$ and let $\mathfrak{M}\left(\widetilde{\Pi}_{j}\right)$ be the space of all symmetric positive definite linear operators $M: \widetilde{\Pi}_{j} \rightarrow \widetilde{\Pi}_{j}$. Consider the map $\mathscr{F}_{j}: \mathfrak{M}\left(\widetilde{\Pi}_{j}\right) \rightarrow \mathfrak{M}\left(\widetilde{\Pi}_{j+1}\right)$ given by $\mathscr{F}_{j}(M)=\tilde{\sigma}_{j+1} M\left(I+\tilde{\lambda}_{j+1} M\right)^{-1} \tilde{\sigma}_{j+1}+\tilde{\widetilde{\psi}}_{j+1}$, where $\tilde{\lambda}_{j}=\left\|\tilde{x}_{j}-\tilde{x}_{j-1}\right\|$ and $\tilde{\sigma}_{j+1}$ is the symmetry with respect to $\widetilde{\Pi}_{j+1}$. It is easy to show (cf. the proof of Theorem 6.2 in [22]) that the map $\mathscr{F}_{k} \circ \mathscr{F}_{k-1} \circ \cdots \circ \mathscr{F}_{1}: \mathfrak{M}\left(\tilde{\Pi}_{1}\right) \rightarrow \mathfrak{M}\left(\tilde{\Pi}_{1}\right)$ has a unique fixed point $\tilde{M}_{1}$. Then $\tilde{M}_{2}=\mathscr{F}_{1}\left(\tilde{M}_{1}\right)$ is the unique fixed point of $\mathscr{F}_{1} \circ \mathscr{F}_{k} \circ \mathscr{F}_{k-1} \circ \cdots \circ \mathscr{F}_{2}$, etc.

Let $m=q k+l$. Introduce the configuration $\alpha_{q, l}$ (cf. the beginning of $\S 6)$. The corresponding map $J_{\alpha_{q, 1}}$ will be denoted briefly by $J_{q}$. Recall that $J_{q}: F_{\alpha_{q, l}} \rightarrow S^{n-1}$ and $F_{\alpha_{q, l}} \subset Z_{\omega}$. It follows by Proposition 6.1 and Corollary 3.3 that there exists a unique $u_{q} \in F_{\alpha_{q},}$ such that $\gamma\left(u_{q}\right)$ is an $(\omega, \theta)$-ray of type $\alpha_{q, l}$. Moreover, $\theta \in \mathscr{R}(\omega)$ implies $u_{q} \in U_{\alpha_{q, 1}}$. Thus $d J_{q}\left(u_{q}\right)$ is well defined and we will examine the asymptotic of $\operatorname{det} d J_{q}\left(u_{q}\right)$ as $q \rightarrow \infty$.

Let $x_{1}, \ldots, x_{m}$ and $y_{1}, \ldots, y_{m}, y_{m+1}, \ldots, y_{m+k}$ be the successive reflection points of $\gamma\left(u_{q}\right)$ and $\gamma\left(u_{q+1}\right)$, respectively. Set $\lambda_{i}=\left\|x_{i-1}-x_{i}\right\|, \lambda_{i}^{\prime}=$ $\left\|y_{i-1}-y_{i}\right\|$ and denote by $\widetilde{\psi}_{i}, \tilde{\psi}_{i}^{\prime}$ the operators related to $\left[x_{i}, x_{i+1}\right]$ and $\left[y_{i}, y_{i+1}\right]$, respectively. Introduce $M_{i}$ and $M_{i}^{\prime}$ by (7.6), where $\sigma_{i}, \sigma_{i}^{\prime}$ are the symmetries with respect to $\Pi_{i}$ and $\Pi_{i}^{\prime}$. Setting

$$
p=[m / 2], \quad t=[p / 2],
$$

we have $2 p \leq m<2 p+1,2 t \leq p<2 t+1$, hence $4 t \leq m<4 t+3$. According to Lemma 6.4 and (6.3), we obtain

$$
\begin{gathered}
\left\|x_{i}-y_{i}\right\|<C \delta^{p-i}, \quad i=1, \ldots, t, \\
\left\|x_{i}-y_{i}\right\|<C \delta^{i}, \quad i=t+1, \ldots, p, \\
\left\|x_{p+i}-y_{p+k+i}\right\|<C \delta^{p-i}, \quad i=1, \ldots, t, \\
\left\|x_{p+i}-y_{p+k+i}\right\|<C \delta^{i}, \quad i=t+1, \ldots, m-p, \\
\left\|y_{v k+j}-\tilde{x}_{j}\right\|<C \delta^{v k+j}, \quad v k+j \leq p+k,
\end{gathered}
$$

where $C>0$ and $0<\delta<1$ depend only on $K, \omega$, and $\theta$. Taking $D=C \delta^{p}$, $a=1 / \delta>1,(7.12)$ becomes

$$
\left\|x_{i}-y_{i}\right\|<D a^{i}, \quad i=1, \ldots, t,
$$

and we are in a position to apply Lemma 7.1 to the sequences $x_{1}, \ldots, x_{t}$ and $y_{1}, \ldots, y_{t}$. Since

$$
\left\|M_{1}-A_{1} M_{1}^{\prime} A_{1}^{-1}\right\|=\left\|\widetilde{\psi}_{1}-A_{1} \widetilde{\psi}_{1}^{\prime} A_{1}^{-1}\right\|<C_{1} D(1+a) a<2 C_{1} C \delta^{p-2},
$$


applying (7.9) for $v=1$ and $a_{1}=1 / \delta$, we get

$$
\begin{gathered}
\left|\ln \operatorname{det}\left(I+\lambda_{i+1} M_{i}\right)-\ln \operatorname{det}\left(I+\lambda_{i+1}^{\prime} M_{i}^{\prime}\right)\right| \\
<D E^{\prime} \delta^{-i}+2(n-1) d b^{2 i-1} C_{1} C \delta^{p-2} \\
<D E^{\prime} \delta_{1}^{p-i}+2(n-1) d C C_{1} \delta_{1}^{p+2 i-3} \\
<F_{1} \delta_{1}^{p-i}, \quad i=1, \ldots, t,
\end{gathered}
$$

where $F_{1}=C E^{\prime}+2(n-1) d C C_{1}$ and $\delta_{1}=\max (\delta, b)<1$.

Observe that (7.12) implies $\left\|x_{i}-y_{i}\right\|<C \delta^{i}, i=1, \ldots, t$. Combining this with (7.13), we can apply Lemma 7.1 for $D=C, a=\delta=a_{1}, v=1$. Then we find

$$
\left|\ln \operatorname{det}\left(I+\lambda_{i+1} M_{i}\right)-\ln \operatorname{det}\left(I+\lambda_{i+1}^{\prime} M_{i}^{\prime}\right)\right|<F_{1} \delta_{1}^{i}, \quad i=1, \ldots, p,
$$

where $F_{1}$ and $\delta_{1}$ are the same as above.

Further, we apply Lemma 7.1 for the sequences $\tilde{x}_{1}, \ldots, \tilde{x}_{p+k}$ and $y_{1}, \ldots$, $y_{p+k}$. By (7.1) and (7.3) we obtain $\left\|M_{1}^{\prime}\right\|=\left\|\tilde{\psi}_{1}^{\prime}\right\| \leq 2 \mu_{2} \kappa^{-1}$ and (7.9) yields

$\left|\ln \operatorname{det}\left(I+\lambda_{p+j+1} M_{p+j}^{\prime}\right)-\ln \operatorname{det}\left(I+\tilde{\lambda}_{p+j+1} \tilde{M}_{p+j}\right)\right|<F_{2} \delta_{1}^{p+j}, \quad j=1, \ldots, k$, where $F_{2}>0$ depends only on $K, \omega$, and $\theta$.

Next, consider the sequences $x_{1}, \ldots, x_{p}$ and $y_{k+1}, \ldots, y_{k+p}$ and denote by $A_{j}^{\prime}: \Pi_{j+k}^{\prime} \rightarrow \Pi_{j}$ the corresponding isometries. Applying Lemma 7.1, we find

$$
\left\|M_{p}-A_{p}^{\prime} M_{p+k}^{\prime} A_{p}^{\prime-1}\right\|<F_{3} \delta_{1}^{p}
$$

with some constant $F_{3}=F_{3}(K, \omega, \theta)>0$. Consider the sequences $x_{p+1}, \ldots$, $x_{p+t}$ and $y_{p+k+1}, \ldots, y_{p+k+t}$. Taking into account (7.14) and (7.20), by Lemma 7.1 for $D=C \delta^{p}, a=1 / \delta=a_{1}$, and $v=p$, we obtain

$\left|\ln \operatorname{det}\left(I+\lambda_{p+j+1} M_{p+j}\right)-\ln \operatorname{det}\left(I+\lambda_{p+k+j+1}^{\prime} M_{p+j+k}^{\prime}\right)\right|<F_{4} \delta_{1}^{p-j}, \quad j=1, \ldots, t$, where $F_{4}$ depends only on $K, \omega$, and $\theta$.

Finally, we apply Lemma 7.1 two more times and find

$$
\begin{gathered}
\left|\ln \operatorname{det}\left(I+\lambda_{p+j+1} M_{p+j}\right)-\ln \operatorname{det}\left(I+\lambda_{p+k+j+1}^{\prime} M_{p+k+j}^{\prime}\right)\right|<F_{5} \delta^{j}, \\
j=t+1, \ldots, m-p, \\
|\ln | \operatorname{det} M_{m}|-\ln | \operatorname{det} M_{m+k}^{\prime}||<F_{6} \delta^{p},
\end{gathered}
$$

where $F_{5}>0$ and $F_{6}>0$ depend only on $K, \omega$, and $\theta$.

Set $F=\max \left\{F_{1}, \ldots, F_{6}\right\}$ and

$$
\tilde{c}=-\sum_{j=1}^{k} \ln \operatorname{det}\left(I+\tilde{\lambda}_{j+1} \tilde{M}_{j}\right)<0 .
$$

By the representations of $d J_{q}\left(u_{q}\right)$ and $d J_{q+1}\left(u_{q+1}\right)$ (cf. (3.10)) we obtain

$$
\ln \left|\operatorname{det} d J_{q+1}\left(u_{q+1}\right)\right|=\ln \left|\operatorname{det} d J_{q}\left(u_{q}\right)\right|-\tilde{c}+\varepsilon_{q, l} \text {, }
$$


where

$$
\begin{aligned}
\varepsilon_{q, l}= & \sum_{i=1}^{p}\left[\ln \operatorname{det}\left(I+\lambda_{i+1}^{\prime} M_{i}^{\prime}\right)-\ln \operatorname{det}\left(I+\lambda_{i+1} M_{i}\right)\right] \\
& +\sum_{i=1}^{k}\left[\ln \operatorname{det}\left(I+\lambda_{p+i+1}^{\prime} M_{p+i}^{\prime}\right)-\ln \operatorname{det}\left(I+\tilde{\lambda}_{p+i+1} \tilde{M}_{p+i}\right)\right] \\
& +\sum_{j=1}^{m-p-1}\left[\ln \operatorname{det}\left(I+\lambda_{p+k+j+1}^{\prime} M_{p+k+j}^{\prime}\right)-\ln \operatorname{det}\left(I+\lambda_{p+j+1} M_{p+j}\right)\right] \\
& +\left[\ln \left|\operatorname{det} M_{m+k}^{\prime}\right|-\ln \left|\operatorname{det} M_{m}\right|\right] .
\end{aligned}
$$

Now (7.17), (7.18), (7.19), (7.21), (7.22), and (7.23) yield

$$
\begin{aligned}
\left|\varepsilon_{q, l}\right| & <F\left(2 \sum_{i=1}^{t} \delta_{1}^{p-i}+\sum_{i=t+1}^{p} \delta_{1}^{i}+\sum_{i=1}^{k} \delta_{1}^{p+i}+\sum_{j=t+1}^{m-p-1} \delta_{1}^{j}+\delta_{1}^{p}\right) \\
& <6 F\left(1-\delta_{1}\right)^{-1} \delta_{1}^{t}<F_{0}^{\prime} \delta_{0}^{k q}
\end{aligned}
$$

with $\delta_{0}=\delta_{1}^{1 / 4}, F_{0}^{\prime}=6 F\left(1-\delta_{1}\right)^{-1} \delta_{1}^{-3 / 4}$.

Let $x_{1}^{\infty}, x_{2}^{\infty}, \ldots$ be the successive reflection points of the ray $\gamma^{\infty}(\omega)$ related to the configuration $\alpha$ and constructed in the previous section. Set $\lambda_{i}^{\infty}=$ $\left\|x_{i-1}^{\infty}-x_{i}^{\infty}\right\|$ and let $M_{i}^{\infty}$ be the corresponding symmetric positive definite linear operator. Set $\beta=\left(i_{1}, \ldots, i_{l}\right)$ and

$$
c_{l}(\omega, \theta)=\ln \left|\operatorname{det} M_{l}^{\infty}\right|+\sum_{i=1}^{l-1} \ln \operatorname{det}\left(I+\lambda_{i+1}^{\infty} M_{i}^{\infty}\right)+\sum_{j=1}^{\infty} \varepsilon_{j, l} .
$$

By (7.25) we obtain

$$
\ln \left|\operatorname{det} d J_{q}\left(u_{q}\right)\right|=-q \tilde{c}+c_{l}(\omega, \theta)+\delta_{q, l}
$$

with $\delta_{q, l}=-\sum_{j=q}^{\infty} \varepsilon_{j, l}+\left[\ln \left|\operatorname{det} d J_{\beta}\left(u_{q}\right)\right|-\ln \left|\operatorname{det} d J_{\beta}\left(x_{0}^{\infty}\right)\right|\right]$. The term in the brackets can be estimated by $F_{0}^{\prime \prime} \delta_{0}^{k q}$ with $F_{0}^{\prime \prime}$ depending only on $K, \omega$, and $\theta$. Therefore, by (7.26) we get

$$
\left|\delta_{q, l}\right|<F_{0}^{\prime} \delta_{0}^{k q}\left(1-\delta_{0}^{k}\right)^{-1}+F_{0}^{\prime \prime} \delta_{0}^{k q}<F_{0} \delta_{0}^{k q} .
$$

Now let $\gamma_{m}^{1 l}$ be the $(\omega, \theta)$-ray with $m$ reflections, introduced in $\S 6$, and let $c_{m}^{1 l}$ be the coefficient in front of $\delta^{(n-1) / 2}\left(t+T_{m}^{1 l}\right)$ in formula (1.5) for the leading singularity at $-T_{m}^{1 l}$. Set $c_{\alpha}=\tilde{c} / 2$. The above considerations lead to

Theorem 7.3. There exist constants $C_{\alpha, \omega, \theta}^{1 l}, 0<\delta_{0}<1$, depending only on $\alpha$, $\omega, \theta$, and $l=1, \ldots, k$ such that

$$
\ln \left|c_{q k+l}^{1 l}\right|=q c_{\alpha}+C_{\alpha, \omega, \theta}^{1 l}+O\left(\delta_{0}^{q}\right), \quad q \rightarrow \infty .
$$

Remark 7.4. It follows easily by our argument in $\S 6$ of [22] that $\mu_{\alpha}=\Pi_{j=1}^{n-1}\left|\mu_{j}\right|=$ $\Pi_{j=1}^{k} \operatorname{det}\left(I+\tilde{\lambda}_{j+1} \tilde{M}_{j}\right),\left|\mu_{j}\right|>1, \mu_{j}, j=1, \ldots, n-1$, being the eigenvalues of 
the (linear) Poincare map related to $\gamma_{\alpha}$. Consequently, from the asymptotics (7.27) we can recover $\mu_{\alpha}$.

\section{APPENDIX}

Here we prove the following lemma, which has been applied in $\S 7$. We use some notation from $\S 7$.

Lemma A. Let $(x, y)$ and $\left(x^{\prime}, y^{\prime}\right)$ be two pairs of points with $x, x^{\prime} \in \partial K_{i}$ and $y, y^{\prime} \in \partial K_{j}, i \neq j$, and let $\varepsilon>0$ be such that $\left\|x-x^{\prime}\right\| \leq \varepsilon$ and $\left\|y-y^{\prime}\right\| \leq \varepsilon$. Assume $\langle e, \nu(x)\rangle \geq \kappa$ and $\left\langle e^{\prime}, \nu\left(x^{\prime}\right)\right\rangle \geq \kappa$ for $e=(y-x) /\|y-x\|$ and $e^{\prime}=\left(y^{\prime}-x^{\prime}\right) /\left\|y^{\prime}-x^{\prime}\right\|$. Let $\tilde{\psi}: \Pi \rightarrow \Pi$ and $\tilde{\psi}^{\prime}: \Pi^{\prime} \rightarrow \Pi^{\prime}$ be the operators related to $[x, y]$ and $\left[x^{\prime}, y^{\prime}\right]$, respectively. Then there exists an isometry $A: \mathbf{R}^{n} \rightarrow \mathbf{R}^{n}$ with $A\left(\Pi^{\prime}\right)=\Pi$ and $\|A-I\|<C_{1} \varepsilon$ such that $\left\|\tilde{\psi}-A \tilde{\psi}^{\prime} A^{-1}\right\|<C_{1} \varepsilon$, where $C_{1}>0$ is a constant depending only on $\kappa$ and $K$.

Proof. Let $A_{1}: \mathbf{R}^{n} \rightarrow \mathbf{R}^{n}$ be the translation determined by $\overrightarrow{x^{\prime}} x$. Set $\nu^{\prime \prime}=$ $A_{1}\left(\nu\left(x^{\prime}\right)\right)$ and denote by $A_{2}$ the rotation with rotation angle

$$
\varphi=\arccos \left\langle\nu(x), \nu\left(x^{\prime}\right)\right\rangle
$$

such that $A_{2}\left(\nu^{\prime \prime}\right)=\nu,\left.A_{2}\right|_{\left\{\nu(x), \nu^{\prime \prime}\right\}^{\perp}}=$ Id. Set $e^{\prime \prime}=A_{2} \circ A_{1}\left(e^{\prime}\right)$ and denote by $A_{3}$ the rotation with $A_{3}\left(e^{\prime \prime}\right)=e$ and $\left.A_{3}\right|_{\left\{e, e^{\prime \prime}\right\}^{\perp}}=\mathrm{Id}$. Set $A=A_{3} \circ A_{2} \circ A_{1}$. It is easy to see now that $\left\|A_{i}-I\right\|<$ const $\varepsilon$ for $i=1,2,3$. For example,

$$
\left\|A_{2}-I\right\|=\left\|\left(\begin{array}{cc}
1-\cos \varphi & \sin \varphi \\
-\sin \varphi & 1-\cos \varphi
\end{array}\right)\right\|=\sqrt{2(1-\cos \varphi)}=\left\|\nu(x)-\nu\left(x^{\prime}\right)\right\|,
$$

and using the smoothness of the Gauss map on $\partial K$ and the compactness of $\partial K$, we find $\left\|\nu(x)-\nu\left(x^{\prime}\right)\right\|<$ const $\left\|x-x^{\prime}\right\|<$ const $\varepsilon$.

Therefore $\|A-I\|<C_{1}^{\prime} \varepsilon$ for some constant $C_{1}^{\prime}>0$.

For convenience set $\tilde{\chi}=A \tilde{\psi}^{\prime} A^{-1}, G=G_{x}, G=G_{x^{\prime}}$. Take $u \in \sqcap$ with $\|u\|=1$ and set $u^{\prime}=A^{-1} u$. Then $u^{\prime} \in \Pi^{\prime}$ and $\left\|u^{\prime}\right\|=1$. Let $\pi: \Pi \rightarrow T_{x} \partial K$ and $\pi^{\prime}: \Pi^{\prime} \rightarrow T_{x^{\prime}} \partial K$ be the projections along $e$ and $e^{\prime}$, respectively, and let $v=\pi u, \quad v^{\prime}=\pi^{\prime} u^{\prime}$. Then we have

$$
\begin{aligned}
& |\langle\tilde{\psi} u, u\rangle-\langle\tilde{\chi} u, u\rangle|=\left|\langle\tilde{\psi} u, u\rangle-\left\langle\tilde{\psi}^{\prime} u^{\prime}, u^{\prime}\right\rangle\right|=\mid 2\langle e, \nu(x)\rangle\langle G \pi u, \pi u\rangle \\
& \quad-2\left\langle e^{\prime}, \nu\left(x^{\prime}\right)\right\rangle\left\langle G^{\prime} \pi^{\prime} u^{\prime}, \pi^{\prime} u^{\prime}\right\rangle|\leq 2|\langle e, \nu(x)\rangle-\left\langle e^{\prime}, \nu\left(x^{\prime}\right)\right\rangle \mid\langle G v, v\rangle \\
& \quad+2\left\langle e^{\prime}, \nu\left(x^{\prime}\right)\right\rangle\left|\langle G v, v\rangle-\left\langle G^{\prime} v^{\prime}, v^{\prime}\right\rangle\right|<\operatorname{const} \varepsilon+2\left|\langle G v, v\rangle-\left\langle G^{\prime} v^{\prime}, v\right\rangle\right| .
\end{aligned}
$$

By the smoothness of the Riemannian metric on $\partial K$ we get $\left|\langle G v, v\rangle-\left\langle G v^{\prime}, v^{\prime}\right\rangle\right|$ $<$ const $\left\|v-v^{\prime}\right\|$. Finally, using $\|\pi\| \leq 1 / \kappa$ and $\left\|\pi^{\prime}\right\| \leq 1 / \kappa$, we find

$$
\left\|v-v^{\prime}\right\|=\left\|\pi u-\pi^{\prime} A^{-1} u\right\| \leq\left\|\pi-\pi^{\prime} A^{-1}\right\|+\left\|\pi^{\prime}\right\|\|A-I\|<\text { const } \varepsilon .
$$

Therefore $|\langle(\tilde{\psi}-\tilde{\chi}) u, u\rangle|<$ const $\varepsilon$ and this yields $\|\tilde{\psi}-\tilde{\chi}\|<C_{1}^{\prime \prime} \varepsilon$ for some constant $C_{1}^{\prime \prime}>0$. $C_{1} \varepsilon$.

Setting $C_{1}=\max \left(C_{1}^{\prime}, C_{1}^{\prime \prime}\right)>0$, we get $\|A-I\|<C_{1} \varepsilon$ and $\left\|\tilde{\psi}-A \tilde{\psi}^{\prime} A^{-1}\right\|<$ 


\section{REFERENCES}

1. C. Bardos, J. C. Guillot, and J. Ralston, La relation de Poisson pour l'équation des ondes dans un ouvert non borné. Application à la théorie de la diffusion, Comm. Partial Differential Equations 7 (1982), 905-958.

2. Y. Colin de Verdiére, Sur les longueurs des trajectories périodiques d'un billiard, Géométrie Symplectique et de Contact: Autour du Théorème de Poincaré-Birkhoff, Hermann, 1984, pp. 122-139.

3. J. J. Duistermaat and V. Guillemin, The spectrum of positive elliptic operators and periodic geodesics, Invent. Math. 29 (1975), 39-79.

4. C. Gérard, Asymptotique des pôles de la matrice de scattering pour deux obstacles strictement convexes, Prépublications de l'Université Paris-Sud, 1986.

5. V. Guillemin, Sojourn time and asymptotic properties of the scattering matrix. Publ. Res. Inst. Math. Sci. 12 (1977), 69-88.

6. V. Guillemin and R. Melrose, The Poisson summation formula for manifolds with boundary, Adv. in Math. 32 (1979), 204-232.

7. M. Ikawa, On the poles of the scattering matrix for two strictly convex obstacles, J. Math. Kyoto Univ. 23 (1983), 127-194.

8. _ Precise information on the poles of the scattering matrix for two strictly convex obstacles, J. Math. Kyoto Univ. 27 (1987), 69-102.

9. __ Decay of solutions of the wave equation in the exterior of several convex bodies, Ann. Inst. Fourier 38 (1988), 113-146.

10. P. Lax and R. Phillips, Scattering theory, Academic Press, New York, 1967.

11. A. Majda, $A$ representation formula for the scattering operator and the inverse problem for arbitrary bodies, Comm. Pure Appl. Math. 30 (1977), 165-194.

12. S. Marvizi and R. Melrose, Spectral invariants and convex planar regions, J. Differential Geometry 17 (1982), 472-502.

13. R. Melrose, Singularities and energy decay of acoustical scattering, Duke Math. J. 46 (1979), 43-59.

14. R. Melrose and J. Sjöstrand, Singularities in boundary value problems. I, II, Comm. Pure Appl. Math. 31 (1978), 593-617 and 35 (1982), 129-168.

15. S. Nakamura and H. Soga, Singularities of the scattering kernel for two balls, J. Math. Soc. Japan 40 (1988), 205-220.

16. V. Petkov, High frequency asymptotics of the scattering amplitude for non-convex bodies, Comm. Partial Differential Equations 5 (1980), 293-329.

17. __ Singularities of the scattering kernel, Nonlinear Partial Differential Equations and their Applications, Collège de France Seminar, VI, Pitman, 1984, pp. 288-296.

18. V. Petkov and P. Vogel, La représentation de l'application de Poincaré correspondant aux rayons périodiques réflechissants, C. R. Acad. Sci. Paris Ser. A 296 (1983), 633-635.

19. V. Petkov and L. Stojanov, Periodic geodesics of generic nonconvex domains in $\mathbf{R}^{2}$ and the Poisson relation, Bull. Amer. Math. Soc. 15 (1986), 88-90.

20. __ Propriétés génériques de l'application de Poincaré et des géodesiques périodiques généralisées, Seminaire Equations aux Dérivées Partielles, Ecole Polytechnique, Exposé XI, 19851986.

21. _ Spectrum of the Poincaré map for periodic reflecting rays in generic domains, Math. $\mathrm{Z}$. 194 (1987), 505-518.

22. _ _ Periods of multiple reflecting geodesics and inverse spectral results, Amer. J. Math. 109 (1987), 619-668.

23. Singularities of the scattering kernel for non-convex obstacles, Conférence Equations aux Dérivées Partielles, Saint-Jean-de-Monts, 1987. 
24. Ya. G. Sinai, Developments of Krylov ideas, An addendum to the book: N. S. Krylov: Works on the Foundations of Statistical Physics, Princeton Univ. Press, 1979.

25. __ Dynamical systems with elastic reflections. Ergodic properties of dispersing billiards, Uspehi Mat. Nauk 25 (1970), 141-192 (Russian).

26. H. Soga, Singularities of the scattering kernel for convex obstacles, J. Math. Kyoto Univ. 22 (1983), 729-765.

27. __ Conditions against rapid decrease of oscillatory integrals and their applications to inverse scattering problems, Osaka J. Math. 23 (1986), 441-456.

28. N. Tchernov, Construction of transversal foliations for multidimensional semi-scattering billiards, Functional Anal. Appl. 16 (1982), 33-46 (Russian).

29. K. Yamamoto, Characterization of a convex obstacle by singularities of the scattering kernel, J. Differential Equations 64 (1986), 283-293.

Institute of Mathematics, Bulgarian ACADemy of Sciences, 1090 Sofia, Bulgaria 